\title{
Ionic modified TBD supported on magnetic nanoparticles: a highly efficient and recoverable catalyst for organic transformations
}

Anguo Ying, ${ }^{\mathrm{a}, *}$ HailiangHou, ${ }^{\mathrm{b}}$ Shuo Liu, ${ }^{\mathrm{c}}$ Gang Chen, ${ }^{\mathrm{a}}$ Jianguo Yang, ${ }^{\mathrm{a}}$ SonglinXu, ${ }^{\mathrm{b}, *}$

( ${ }^{a}$ School of Pharmaceutical and Chemical Engineering, Taizhou University, Taizhou 318000,

China)

( ${ }^{\mathrm{b}}$ School of Chemical Engineering and Technology, Tianjin University, Tianjin 300072, China)

( ${ }^{c}$ College of Chemistry, Nankai University, Tianjin 300071, China)

1.Optimization reaction conditions of aza-Michael addition of imidazole to methyl acrylate......2

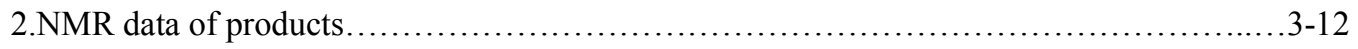

3.NMR spectrum of products......................................................

* Corresponding authors. Tel/fax: +8657688660359.

E-mail address: yinganguo@163.com or agying@tzc.edu.cn (A. Ying), slxu@tju.edu.cn (S. Xu). 
Table S1 Evaluation of the effects of the catalyst and solvent on the aza-Michael addition ${ }^{\mathrm{a}}$

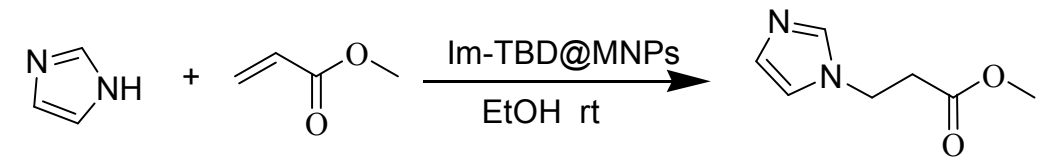

\begin{tabular}{ccccc}
\hline Entry & Catalyst $(\mathrm{mg})$ & Solvent $(3 \mathrm{~mL})$ & Time $(\mathrm{h})$ & Yield $(\%)^{[\mathrm{b}]}$ \\
\hline 1 & Im-TBD@MNPs (40) & Ethanol & 2 & 92 \\
2 & TBD @MNPs (60) & Ethanol & 2 & 67 \\
3 & MNPs (100) & Ethanol & 6 & Trace \\
4 & No catalyst & Ethanol & 24 & Trace \\
5 & Im-TBD@MNPs (100) & Ethanol & 2 & 93 \\
6 & Im-TBD@MNPs (30) & Ethanol & 2 & 70 \\
7 & Im-TBD@MNPs (10) & Ethanol & 2 & 40 \\
8 & Im-TBD@MNPs (40) & Solvent Free & 2 & 86 \\
9 & Im-TBD@MNPs (40) & Water & 2 & 69 \\
10 & Im-TBD@MNPs (40) & Toluene & 2 & 80 \\
\hline
\end{tabular}

${ }^{\mathrm{a}}$ imidazole $(2.0 \mathrm{mmol})$, methyl acrylate $(2.2 \mathrm{mmol})$, room temperature.

${ }^{\mathrm{b}}$ Isolated yield based onimidazole 


\section{1,3-diphenylurea (Table 2, entry 1)}<smiles>O=C(Nc1ccccc1)Nc1ccccc1</smiles>

${ }^{1} \mathrm{H}$ NMR ( $400 \mathrm{MHz}, \mathrm{DMSO}$ ) (ppm): $\delta 6.96$ (t, 2H, $J=7.2 \mathrm{~Hz}$ ), 7.28 (t, 4H, $\left.J=7.2 \mathrm{~Hz}\right), 7.44$ (d, 4H, $J=7.6 \mathrm{~Hz}$ ), 8.64 (s, 2H); ${ }^{13} \mathrm{C}$ NMR ( $\left.100 \mathrm{MHz}, \mathrm{DMSO}\right): \delta 123.42,127.02,133.99,144.93,157.75$. MS m/z: 212.09, Anal. Calcd for $\mathrm{C}_{13} \mathrm{H}_{12} \mathrm{~N}_{2} \mathrm{O}: \mathrm{C}, 73.56 ; \mathrm{H}, 5.70 ; \mathrm{N}, 13.20 ; \mathrm{O}, 7.54$; found $\mathrm{C}, 73.50$; $\mathrm{H}, 5.74 ; \mathrm{N}, 13.18 ; \mathrm{O}, 7.58$.

\section{1,3-bis(4-methoxyphenyl)urea (Table 2, entry 2)}

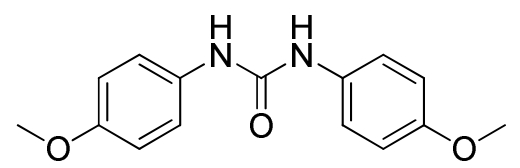

${ }^{1} \mathrm{H}$ NMR ( $400 \mathrm{MHz}, \mathrm{DMSO}$ ) (ppm): $\delta 3.71$ (s, 6H), 6.84 (d, 4H, $\left.J=9.2 \mathrm{~Hz}\right), 7.32$ (d, 4H, $J=9.2 \mathrm{~Hz}$ ), 8.36 (s, 2H); ${ }^{13} \mathrm{C}$ NMR ( $\left.100 \mathrm{MHz}, \mathrm{DMSO}\right): \delta 55.65,114.44,120.39,133.42,153.42,154.82 . \mathrm{MS}$ m/z: 272.12, Anal. Calcd for $\mathrm{C}_{15} \mathrm{H}_{16} \mathrm{~N}_{2} \mathrm{O}_{3}: \mathrm{C}, 66.16 ; \mathrm{H}, 5.92 ; \mathrm{N}, 10.29 ; \mathrm{O}, 17.63$; found $\mathrm{C}, 66.10 ; \mathrm{H}$, $5.95 ; \mathrm{N}, 10.27 ; \mathrm{O}, 17.68$.

\section{1,3-di-o-tolylurea (Table 2, entry 3)}

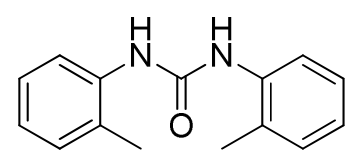

${ }^{1} \mathrm{H}$ NMR ( $400 \mathrm{MHz}, \mathrm{DMSO}$ ) (ppm): $\delta 2.27$ (s, 6H), 6.94 (t, 2H, $\left.J=7.2 \mathrm{~Hz}\right), 7.17$ (d, 2H, $J=7.6 \mathrm{~Hz}$ ), 7.14 (t, $2 \mathrm{H}, J=8.0 \mathrm{~Hz}), 7.80$ (d, 2H, $J=8.0 \mathrm{~Hz}), 8.24$ (s, 2H); ${ }^{13} \mathrm{C}$ NMR ( $\left.100 \mathrm{MHz}, \mathrm{DMSO}\right): \delta 23.24$, $126.75,127.91,131.30,133.00,135.39,142.73,158.19$. MS m/z: 240.13, Anal. Calcd for $\mathrm{C}_{15} \mathrm{H}_{16} \mathrm{~N}_{2} \mathrm{O}: \mathrm{C}, 74.97 ; \mathrm{H}, 6.71 ; \mathrm{N}, 11.66 ; \mathrm{O}, 6.66 ; \mathrm{O}, 15.98$; found $\mathrm{C}, 74.94 ; \mathrm{H}, 6.73 ; \mathrm{N}, 11.62 ; \mathrm{O}$, 
6.71.

\section{1,3-di-m-tolylurea (Table 2, entry 4)}

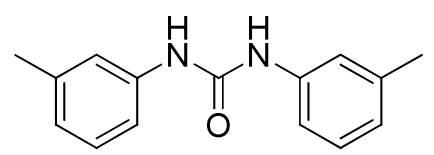

${ }^{1} \mathrm{H}$ NMR ( $400 \mathrm{MHz}, \mathrm{DMSO}$ ) (ppm): $\delta 2.28$ (s, 6H), 6.77 (d, 2H, $\left.J=7.6 \mathrm{~Hz}\right), 7.15$ (t, 2H, $J=8.0 \mathrm{~Hz}$ ), 7.21 (d, 2H, $J=8.0 \mathrm{~Hz}), 7.31$ (s, 2H), 8.56 (s, 2H); ${ }^{13} \mathrm{C}$ NMR ( $\left.100 \mathrm{MHz}, \mathrm{DMSO}\right): \delta 26.43,120.56$, 123.90, 127.73, 133.82, 143.15, 144.88, 157.71. MS m/z: 240.13, Anal. Calcd for $\mathrm{C}_{15} \mathrm{H}_{16} \mathrm{~N}_{2} \mathrm{O}: \mathrm{C}$, 74.97; H, 6.71; N, 11.66; O, 6.66; O, 15.98; found C, 74.94; H, 6.73; N, 11.62; O, 6.71.

\section{1,3-di-p-tolylurea (Table 2, entry 5)}

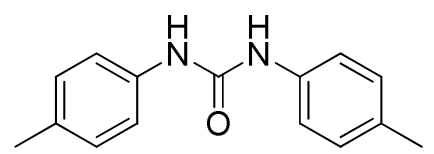

${ }^{1} \mathrm{H}$ NMR ( $400 \mathrm{MHz}, \mathrm{DMSO}$ ) (ppm): $\delta 2.24$ (s, 6H), 7.06 (d, 4H, $\left.J=8.0 \mathrm{~Hz}\right), 7.34$ (d, 4H, $J=8.4 \mathrm{~Hz}$ ), 8.49 (s, 2H); ${ }^{13} \mathrm{C}$ NMR ( $\left.100 \mathrm{MHz}, \mathrm{DMSO}\right): \delta 20.79,118.70,129.61,130.96,137.70,153.10 . \mathrm{MS}$ m/z: 240.13, Anal. Calcd for $\mathrm{C}_{15} \mathrm{H}_{16} \mathrm{~N}_{2} \mathrm{O}: \mathrm{C}, 74.97 ; \mathrm{H}, 6.71 ; \mathrm{N}, 11.66 ; \mathrm{O}, 6.66$; found $\mathrm{C}, 74.94 ; \mathrm{H}$, $6.73 ; \mathrm{N}, 11.62 ; \mathrm{O}, 6.71$.

\section{1,3-bis(4-ethoxyphenyl)urea (Table 2, entry 6)}

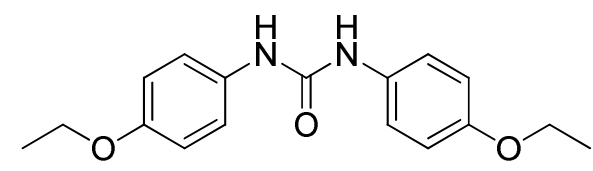

${ }^{1} \mathrm{H}$ NMR ( $\left.400 \mathrm{MHz}, \mathrm{DMSO}\right)(\mathrm{ppm}): \delta 1.30$ (t, 6H, J=7.2Hz), 3.94-3.99 (m, 4H), $6.82(\mathrm{~d}, 4 \mathrm{H}$, $J=8.8 \mathrm{~Hz}), 7.31$ (d, 4H, $J=8.8 \mathrm{~Hz}), 8.35(\mathrm{~s}, 2 \mathrm{H}) ;{ }^{13} \mathrm{C}$ NMR ( $\left.100 \mathrm{MHz}, \mathrm{DMSO}\right): \delta 15.20,63.58$, 115.03, 120.37, 133.34, 153.42, 154.05. MS bm/z: 300.15, Anal. Calcd for $\mathrm{C}_{17} \mathrm{H}_{20} \mathrm{~N}_{2} \mathrm{O}_{3}: \mathrm{C}, 67.98$; 


\section{Methyl-3-(1H-imidazol-1-yl)propanoate (Table 3, entry 1)}

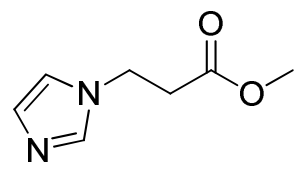

${ }^{1} \mathrm{H}$ NMR (400 MHz, $\mathrm{CDCl}_{3}$ ) (ppm): $\delta 2.72(\mathrm{t}, 2 \mathrm{H}, J=6 \mathrm{~Hz}), 3.62(\mathrm{~s}, 3 \mathrm{H}), 4.20(\mathrm{t}, 2 \mathrm{H}, J=2.4 \mathrm{~Hz}), 6.86(\mathrm{~d}$, $1 \mathrm{H}, J=4.4 \mathrm{~Hz}), 6.95(\mathrm{~d}, 1 \mathrm{H}, J=6 \mathrm{~Hz}), 7.43(\mathrm{~d}, 1 \mathrm{H}, J=4.8 \mathrm{~Hz}) ;{ }^{13} \mathrm{C} \mathrm{NMR}\left(100 \mathrm{MHz}, \mathrm{CDCl}_{3}\right)(\mathrm{ppm}): \delta$ 35.46, 42.00, 51.77, 118.75, 129.17, 137.10, 170.84. MS m/z=154.1, Anal Calcd for $\mathrm{C}_{7} \mathrm{H}_{10} \mathrm{~N}_{2} \mathrm{O}_{2}: \mathrm{C}$, 54.54; H, 6.54; N, 18.17; O, 20.75; found C, 54.50; H, 6.58; N, 18.14; O,20.78.

\section{Methyl-3-(4-nitro-1H-imidazol-1-yl)propanoate (Table 3, entry 2)}<smiles>COC(=O)CCn1cnc([N+](=O)[O-])c1</smiles>

${ }^{1} \mathrm{H}$ NMR (400 MHz, $\mathrm{CDCl}_{3}$ ) (ppm): $\delta 2.88(\mathrm{t}, 2 \mathrm{H}, J=6 \mathrm{~Hz}), 3.73(\mathrm{~s}, 3 \mathrm{H}), 4.38(\mathrm{t}, 2 \mathrm{H}, J=6 \mathrm{~Hz}), 7.53(\mathrm{~s}$,

1H), 7.87(s, 1H); ${ }^{13} \mathrm{C} \mathrm{NMR}\left(100 \mathrm{MHz}, \mathrm{CDCl}_{3}\right)(\mathrm{ppm}): \delta 35.1,43.5,52.5,119.5,136.4,148.2,170.5$. MS m/z=199.1, Anal Calcd for $\mathrm{C}_{7} \mathrm{H}_{9} \mathrm{~N}_{3} \mathrm{O}_{4}: \mathrm{C}, 42.21 ; \mathrm{H}, 4.55 ; \mathrm{N}, 21.10 ; \mathrm{O}, 32.13$; found $\mathrm{C}, 42.10 ; \mathrm{H}$, $4.62 ; \mathrm{N}, 21.08 ; \mathrm{O}, 32.20$.

3-(2-Isopropylimidazolyl)-propionic acid methyl ester (Table 3, entry 3):<smiles>COC(=O)CCn1ccnc1C(C)C</smiles> 
${ }^{1} \mathrm{H}$ NMR (400 MHz, $\mathrm{CDCl}_{3}$ ) (ppm): 6.94 (d, 1H, J=1.2 Hz, imidazolyl), $6.81(\mathrm{~d}, 1 \mathrm{H}, \mathrm{J}=1.2 \mathrm{~Hz}$, imidazolyl), 4.20 (t, 2H, J=7.2 Hz, $\mathrm{CH}_{2}$ ), 3.71 (s, 3H, $\mathrm{OCH}_{3}$ ), 3.03 (q, 1H, J=7.2 Hz, CH) 2.76 (t, $\left.2 \mathrm{H}, \mathrm{J}=7.2 \mathrm{~Hz}, \mathrm{CH}_{2}\right), 1.33\left(\mathrm{~d}, 6 \mathrm{H}, 2 \mathrm{CH}_{3}\right) ;{ }^{13} \mathrm{C} \mathrm{NMR}\left(100 \mathrm{MHz}, \mathrm{CDCl}_{3}\right)(\mathrm{ppm}):$ 170.9, 152.4, 127.4, $118.3,52.0,40.6,35.6,25.7,21.8$.

\section{Ethyl-3-(4-methyl-1H-imidazol-1-yl)propanoate (Table 3, entry 4)}

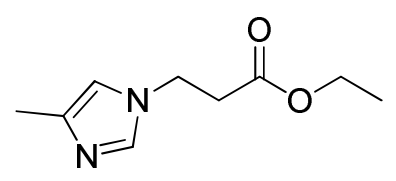

${ }^{1} \mathrm{H}$ NMR $\left(400 \mathrm{MHz}, \mathrm{CDCl}_{3}\right)(\mathrm{ppm}): \delta 0.97(\mathrm{t}, 3 \mathrm{H}, J=6.8 \mathrm{~Hz}), 1.95(\mathrm{~s}, 3 \mathrm{H}), 2.47(\mathrm{t}, 2 \mathrm{H}, J=6.8 \mathrm{~Hz})$ 3.84-3.90(m, 4H), 6.40(s, $1 \mathrm{H}), 7.12(\mathrm{~s}, 1 \mathrm{H}) .{ }^{13} \mathrm{C} \mathrm{NMR}\left(100 \mathrm{MHz}, \mathrm{CDCl}_{3}\right): \delta 13.84,14.00,35.71$, 41.93, 60.69, 115.06, 136.17, 138.1, 170.4. MS m/z=182.1, Anal Calcd for $\mathrm{C}_{9} \mathrm{H}_{14} \mathrm{~N}_{2} \mathrm{O}_{2}: \mathrm{C}, 59.32 ; \mathrm{H}$, 7.74; N, 15.37; O, 17.56; found C, 59.27; H, 7.78; N, 15.35; O, 17.60 .

\section{Butyl-3-(1H-imidazol-1-yl)propanoate (Table 3, entry 5)}

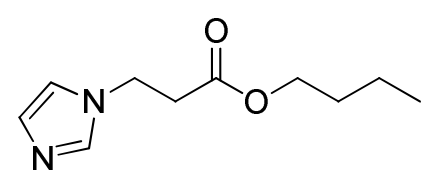

${ }^{1} \mathrm{H}$ NMR (400 MHz, $\mathrm{CDCl}_{3}$ ) (ppm): $\delta$ 0.76(t, 3H, $\left.J=7.6 \mathrm{~Hz}\right), 1.18(\mathrm{~d}, 2 \mathrm{H}, J=7.6 \mathrm{~Hz}), 1.41(\mathrm{~d}, 2 \mathrm{H}$, $J=7.6 \mathrm{~Hz}), 2.62(\mathrm{t}, 2 \mathrm{H}, J=6.8 \mathrm{~Hz}), 3.93(\mathrm{t}, 2 \mathrm{H}, J=6.4 \mathrm{~Hz}), 4.11(\mathrm{t}, 2 \mathrm{H}, J=6.8 \mathrm{~Hz}), 6.80(\mathrm{~s}, 1 \mathrm{H}), 6.87(\mathrm{~s}$, 1H), 7.35(s, 1H); ${ }^{13} \mathrm{C} \mathrm{NMR}\left(100 \mathrm{MHz}, \mathrm{CDCl}_{3}\right)(\mathrm{ppm}): \delta 14.1,21.8,62.5,101.4,115.7,128.8,131.2$ 144.6, 155.0, 162.7. MS m/z=196.1, Anal Calcd for $\mathrm{C}_{10} \mathrm{H}_{16} \mathrm{~N}_{2} \mathrm{O}_{2}$ : C, 61.20; H, 8.22; N, 14.27; O16.31; found C, 61.14; H, 8.26; N, 14.25; O, 16.35. 
Butyl-3-(1H-imidazol-1-yl)butanoate (Table 3, entry 6)

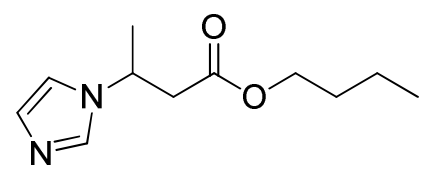

${ }^{1} \mathrm{H}$ NMR $\left(400 \mathrm{MHz}, \mathrm{CDCl}_{3}\right)(\mathrm{ppm}): \delta 0.87(\mathrm{t}, 3 \mathrm{H}, J=7.6 \mathrm{~Hz}), 1.16(\mathrm{~d}, 3 \mathrm{H}, J=7.2 \mathrm{~Hz}), 1.26-1.35(\mathrm{~m}$, $2 \mathrm{H}), 1.51-1.59(\mathrm{~m}, 2 \mathrm{H}), 2.81-2.90(\mathrm{~m}, 1 \mathrm{H}), 3.96-4.03(\mathrm{~m}, 1 \mathrm{H}), 4.04-4.07(\mathrm{~m}, 2 \mathrm{H}), 4.20-4.25(\mathrm{~m}, 1 \mathrm{H})$, 6.89(s, 1H), 7.02(s, 1H), 7.45(s, 1H). ${ }^{13} \mathrm{C}$ NMR (100 MHz, $\left.\mathrm{CDCl}_{3}\right)(\mathrm{ppm}): \delta 13.55,14.84,18.92$, $30.38,41.40,49.16,64.84,119.22,129.18,137.44,173.72 . \mathrm{MS} \mathrm{m} / \mathrm{z}=210.1$, Anal Calcd for $\mathrm{C}_{11} \mathrm{H}_{18} \mathrm{~N}_{2} \mathrm{O}_{2}: \mathrm{C}, 62.83 ; \mathrm{H}, 8.63 ; \mathrm{N}, 13.32 ; \mathrm{O}, 15.22$; found $\mathrm{C}, 62.76 ; \mathrm{H}, 8.66 ; \mathrm{N}, 13.31 ; \mathrm{O}, 15.27$.

3-(4-nitro-1H-imidazol-1-yl)propanenitrile (Table 3, entry 7)

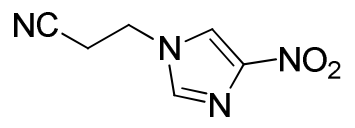

${ }^{1} \mathrm{H}$ NMR $\left(400 \mathrm{MHz}, \mathrm{CDCl}_{3}\right)(\mathrm{ppm}): \delta 3.19(\mathrm{t}, 2 \mathrm{H}, J=6.4 \mathrm{~Hz}), 4.31(\mathrm{t}, 2 \mathrm{H}, J=6.4 \mathrm{~Hz}), 7.95(\mathrm{~d}, 1 \mathrm{H}$, $J=1.6 \mathrm{~Hz}), 8.50(\mathrm{~d}, 1 \mathrm{H}, J=1.6 \mathrm{~Hz}) ;{ }^{13} \mathrm{C} \mathrm{NMR}\left(100 \mathrm{MHz}, \mathrm{CDCl}_{3}\right)(\mathrm{ppm}): \delta 19.4,43.5,118.5,121.7$, 138.0, 147.6. MS m/z=166.1, Anal Calcd for $\mathrm{C}_{6} \mathrm{H}_{6} \mathrm{~N}_{4} \mathrm{O}_{2}: \mathrm{C}, 43.38 ; \mathrm{H}, 3.64 ; \mathrm{N}, 33.72 ; \mathrm{O}, 19.26$; found $\mathrm{C}, 43.30 ; \mathrm{H}, 3.70 ; \mathrm{N}, 33.70 ; \mathrm{O}, 19.30$.

Methyl-3-(1H-benzo[d]imidazol-1-yl)propanoate (Table 3, entry 8)

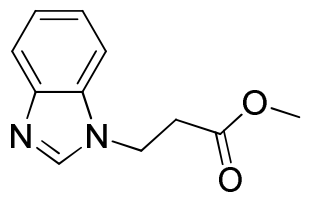


${ }^{1} \mathrm{H}$ NMR (400 MHz, $\mathrm{CDCl}_{3}$ ) (ppm): $\delta 2.53(\mathrm{t}, 2 \mathrm{H}, J=6.4 \mathrm{~Hz}), 3.32(\mathrm{~s}, 3 \mathrm{H}), 4.11(\mathrm{t}, 2 \mathrm{H}, J=6.4 \mathrm{~Hz})$, 7.00-7.03(m, 1H), 7.58-7.59(m, 1H), 7.72(s, 1H); ${ }^{13} \mathrm{C}$ NMR (100 MHz, $\left.\mathrm{CDCl}_{3}\right)(\mathrm{ppm}): \delta 33.78$, $40.00,51.69,109.38,119.99,121.95,122.75,133.22,143.34,143.53,170.89 . \mathrm{MS} \mathrm{m} / \mathrm{z}=204.1$,Anal Calcd for $\mathrm{C}_{11} \mathrm{H}_{12} \mathrm{~N}_{2} \mathrm{O}_{2}$ : C, 64.69; H, 5.92; N, 13.72; O, 15.67; found $\mathrm{C}, 64.61 ; \mathrm{H}, 5.97 ; \mathrm{N}, 13.70 ; \mathrm{O}$, 15.72 .

\section{Methyl-3-morpholinopropanoate (Table 4, entry 1)}

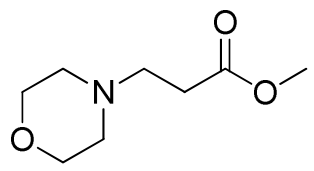

${ }^{1} \mathrm{H}$ NMR (400 MHz, $\left.\mathrm{CDCl}_{3}\right)(\mathrm{ppm}): 2.42(\mathrm{t}, 4 \mathrm{H}, J=4.8 \mathrm{~Hz}), 2.48(\mathrm{t}, 2 \mathrm{H}, J=6.4 \mathrm{~Hz}), 2.65(\mathrm{t}, 2 \mathrm{H}$, $J=7.2 \mathrm{~Hz}), 3.66(\mathrm{t}, 7 \mathrm{H}, J=2.8 \mathrm{~Hz}) ;{ }^{13} \mathrm{C}$ NMR $\left(100 \mathrm{MHz}, \mathrm{CDCl}_{3}\right)(\mathrm{ppm}): 31.62,51.47,53.15,53.69$, 66.65, 172.62. MS m/z=173.1, Anal Calcd for $\mathrm{C}_{8} \mathrm{H}_{15} \mathrm{NO}_{3}$ : C, 55.47; H, 8.73; N, 8.09; O, 27.71; found $\mathrm{C}, 55.42 ; \mathrm{H}, 8.79 ; \mathrm{N}, 8.08 ; \mathrm{O}, 27.71$.

\section{3-(1-Morpholinyl)-propionic acid ethyl ester (Table 4, entry 2)}

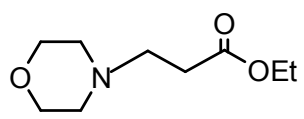

${ }^{1} \mathrm{H}$ NMR (400 MHz, $\left.\mathrm{CDCl}_{3}\right)(\mathrm{ppm}): 4.15\left(\mathrm{q}, 2 \mathrm{H}, \mathrm{J}=7.2 \mathrm{~Hz}, \mathrm{OCH}_{2}\right), 3.7(\mathrm{~m}, 4 \mathrm{H}, \mathrm{J}=4.4 \mathrm{~Hz}$, morpholinyl), 2.69 (t, 2H, J=7.6 Hz, $\mathrm{CH}_{2}$ ), 2.50 (t, 2H, J=7.6 Hz, $\mathrm{CH}_{2}$ ), 2.47 (q, 4H, J=4.4 Hz, morpholinyl), 1.27 (t, 3H, J=7.2 Hz, $\left.\mathrm{CH}_{3}\right) ;{ }^{13} \mathrm{C} \mathrm{NMR}\left(100 \mathrm{MHz}, \mathrm{CDCl}_{3}\right)$ (ppm): 175.4, 66.9, 60.4, $53.9,53.4,32.1,14.2$. 


\section{Butyl-3-morpholinopropanoate (Table 4, entry 3)}

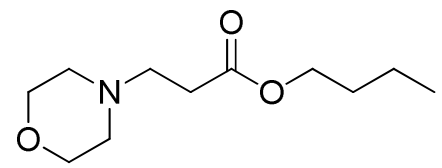

${ }^{1} \mathrm{H}$ NMR (400 MHz, $\mathrm{CDCl}_{3}$ ) (ppm): $\delta$ 0.94(t, 3H, $\left.J=7.6 \mathrm{~Hz}\right) 1.34-1.43(\mathrm{~m}, 2 \mathrm{H}), 1.58-1.65(\mathrm{~m}, 2 \mathrm{H})$, $2.45-2.48(\mathrm{~m}, 4 \mathrm{H}), 2.50(\mathrm{~d}, 2 \mathrm{H}, J=7.2 \mathrm{~Hz}), 2.67-2.70(\mathrm{~m}, 2 \mathrm{H}), 3.70(\mathrm{t}, 4 \mathrm{H}, J=4.8 \mathrm{~Hz}), 4.10(\mathrm{t}, 2 \mathrm{H}$, $J=6.8 \mathrm{~Hz}) .{ }^{13} \mathrm{C} \mathrm{NMR}\left(100 \mathrm{MHz}, \mathrm{CDCl}_{3}\right)(\mathrm{ppm}): \delta 13.7,19.1,30.6,32.1,53.3,54.0,64.3,66.9,172.5$. MS m/z=215.2, Anal Calcd for $\mathrm{C}_{11} \mathrm{H}_{21} \mathrm{NO}_{3}: \mathrm{C}, 61.37 ; \mathrm{H}, 9.83 ; \mathrm{N}, 6.51 ; \mathrm{O}, 22.29$; found $\mathrm{C}, 61.30 ; \mathrm{H}$, $9.89 ; \mathrm{N}, 6.48 ; \mathrm{O}, 22.33$.

\section{3-(1-Morpholinyl)-propanamide(Table 4, entry 4)}

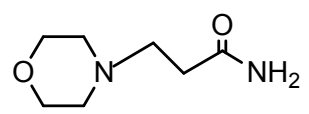

${ }^{1} \mathrm{H}$ NMR (400 MHz, $\left.\mathrm{CDCl}_{3}\right)(\mathrm{ppm}): 7.84(\mathrm{br}, 1 \mathrm{H}, \mathrm{NH}), 6.06(\mathrm{br}, 1 \mathrm{H}, \mathrm{NH}), 3.73(\mathrm{~s}, 4 \mathrm{H}$, morpholinyl), 2.64 (t, 2H, J=6 Hz, $\mathrm{CH}_{2}$ ), 2.51 (s, 4H, morpholinyl), $2.41\left(\mathrm{t}, 2 \mathrm{H}, \mathrm{J}=6 \mathrm{~Hz}, \mathrm{CH}_{2}\right) ;{ }^{13} \mathrm{C}$ $\operatorname{NMR}\left(100 \mathrm{MHz}, \mathrm{CDCl}_{3}\right)(\mathrm{ppm}):$ 175.0, 66.7, 54.1, 52.9, 31.6.

\section{N-(hydroxymethyl)-3-morpholinopropanamide (Table 4, entry 5)}<smiles>O=C(CCN1CCOCC1)NCO</smiles>

${ }^{1} \mathrm{H}$ NMR (400 MHz, $\mathrm{CDCl}_{3}$ ) (ppm): $\delta 2.41(\mathrm{~d}, 2 \mathrm{H}, J=6.4 \mathrm{~Hz}), 2.49(\mathrm{~s}, 4 \mathrm{H}), 2.62(\mathrm{~d}, 2 \mathrm{H}, J=6.4 \mathrm{~Hz})$, 3.69-3.71(m, 4H), 4.69-4.71(m, 2H), 8.71(s, 1H); ${ }^{13} \mathrm{C} \mathrm{NMR}\left(100 \mathrm{MHz}, \mathrm{CDCl}_{3}\right)(\mathrm{ppm}): \delta 32.2,53.0$, 54.1, 63.7, 66.6, 173.7. MS m/z=188.1, Anal Calcd for $\mathrm{C}_{8} \mathrm{H}_{16} \mathrm{~N}_{2} \mathrm{O}_{3}: \mathrm{C}, 51.05 ; \mathrm{H}, 8.57 ; \mathrm{N}, 14.88 ; \mathrm{O}$, 
N,N-dimethyl-3-morpholinopropanamide (Table 4, entry 6)<smiles>CN(C)C(=O)CCN1CCOCC1</smiles>

${ }^{1} \mathrm{H} \mathrm{NMR}\left(400 \mathrm{MHz}, \mathrm{CDCl}_{3}\right)(\mathrm{ppm}): \delta 2.50-2.56(\mathrm{~m}, 6 \mathrm{H}), 2.71-2.75(\mathrm{~m}, 2 \mathrm{H}), 2.95(\mathrm{~s}, 3 \mathrm{H}), 3.03(\mathrm{~s}, 3 \mathrm{H})$, 3.72(t, 4H, $J=4.0 \mathrm{~Hz}) ;{ }^{13} \mathrm{C} \mathrm{NMR}\left(100 \mathrm{MHz}, \mathrm{CDCl}_{3}\right)(\mathrm{ppm}): \delta 30.7,35.4,37.3,53.7,54.5,66.9,171.5$. MS m/z=186.1,AnalCalcd for $\mathrm{C}_{9} \mathrm{H}_{18} \mathrm{~N}_{2} \mathrm{O}_{2}: \mathrm{C}, 58.04 ; \mathrm{H}, 9.74 ; \mathrm{N}, 15.04 ; \mathrm{O}, 17.18$; found C, 57.98; H, 9.79; N, 15.03; O, 17.20.

\section{3-(4-ethylpiperazin-1-yl)-1-morpholinopropan-1-one (Table 4, entry 7)}

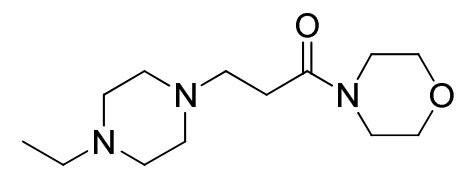

${ }^{1} \mathrm{H}$ NMR (400 MHz, $\mathrm{CDCl}_{3}$ ) (ppm): 3.66-3.67(m, 4H),3.61-3.63(m, 2H), 3.47-3.49(m, 2H), 2.74(d, $2 \mathrm{H}, J=8.0 \mathrm{~Hz}), 2.52-2.55(\mathrm{~m}, 8 \mathrm{H}), 2.39-2.45(\mathrm{~m}, 4 \mathrm{H}), 1.09(\mathrm{t}, 3 \mathrm{H}, J=7.2 \mathrm{~Hz}) ;{ }^{13} \mathrm{C} \mathrm{NMR}(100 \mathrm{MHz}$, $\mathrm{CDCl}_{3}$ ) (ppm): 170.4, 66.9, 66.7, 54.0, 53.2, 52.7, 52.3, 46.0, 41.9, 30.7, 12.0.Anal. Calcd for $\mathrm{C}_{13} \mathrm{H}_{25} \mathrm{~N}_{3} \mathrm{O}_{2}$ : C, 61.15; H, 9.87; N, 16.46; O, 12.53. Found: C, 61.05; H, 9.97; N, 16.38; O, 12.60.

3-(4-(2-hydroxyethyl)piperazin-1-yl)-1-morpholinopropan-1-one (Table 4, entry 8)

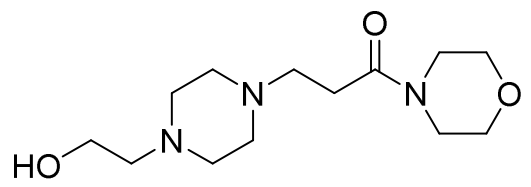

${ }^{1} \mathrm{H}$ NMR (400 MHz, $\left.\mathrm{CDCl}_{3}\right)(\mathrm{ppm}): 3.61-3.69(\mathrm{~m}, 8 \mathrm{H}), 3.47-3.49(\mathrm{~m}, 2 \mathrm{H}), 2.72-2.76(\mathrm{~m}, 4 \mathrm{H})$, 2.51-2.56(m, 10H); ${ }^{13} \mathrm{C}$ NMR (100 $\left.\mathrm{MHz}, \mathrm{CDCl}_{3}\right)(\mathrm{ppm}):$ 170.3, 66.9, 66.6, 59.3, 57.8, 53.9, 53.2, 52.8, 46.0, 41.9, 30.7.Anal.Calcd for $\mathrm{C}_{13} \mathrm{H}_{25} \mathrm{~N}_{3} \mathrm{O}_{3}$ : C, 57.54; H, 9.29; N, 15.49; O, 17.69. Found: C, 
3-(4-ethylpiperazin-1-yl)-N-(2-methyl-4-oxopentan-2-yl)propanamide (Table 4, entry 9)<smiles>CCN1CCN(CCC(=O)NC(C)(C)CC(C)=O)CC1</smiles>

${ }^{1} \mathrm{H}$ NMR (400 MHz, $\left.\mathrm{CDCl}_{3}\right)(\mathrm{ppm}): 8.69(\mathrm{~s}, 1 \mathrm{H}), 3.02(\mathrm{~s}, 2 \mathrm{H}), 2.41-2.60($ overlapped, 12H), $2.25-2.28(\mathrm{~m}, 2 \mathrm{H}), 2.10(\mathrm{~s}, 3 \mathrm{H}), 1.37(\mathrm{~s}, 6 \mathrm{H}), 1.10(\mathrm{t}, 3 \mathrm{H}, J=7.2 \mathrm{~Hz}) ;{ }^{13} \mathrm{C} \mathrm{NMR}\left(100 \mathrm{MHz}, \mathrm{CDCl}_{3}\right)$ (ppm): 207.6, 172.1, 53.9, 52.9, 52.3, 52.2, 51.2, 50.5, 32.8, 31.6, 27.9, 11.9. Anal. Calcd for $\mathrm{C}_{15} \mathrm{H}_{29} \mathrm{~N}_{3} \mathrm{O}_{2}$ : C, 63.57; H, 10.31; N, 14.83; O, 11.29. Found: C, 63.45; H, 10.44; N, 14.77; O, 11.34.

\section{3-(4-methylpiperidin-1-yl)-1-morpholinopropan-1-one (Table 4, entry 10)}

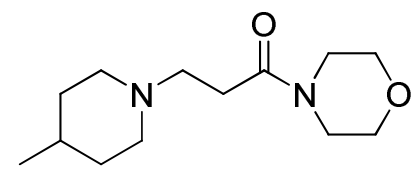

${ }^{1} \mathrm{H} \mathrm{NMR}\left(400 \mathrm{MHz}, \mathrm{CDCl}_{3}\right)(\mathrm{ppm}): 3.66(\mathrm{~d}, 4 \mathrm{H}, J=4.7 \mathrm{~Hz}), 3.62(\mathrm{~d}, 2 \mathrm{H}, J=4.7 \mathrm{~Hz}), 3.47-3.49(\mathrm{~m}, 2 \mathrm{H})$, $2.89(\mathrm{~d}, 2 \mathrm{H}, J=11.3 \mathrm{~Hz}), 2.68-2.71(\mathrm{~m}, 2 \mathrm{H}), 2.52-2.56(\mathrm{~m}, 2 \mathrm{H}) 2.00(\mathrm{t}, 2 \mathrm{H}, J=11.2 \mathrm{~Hz}), 1.64(\mathrm{~d}, 2 \mathrm{H}$, $J=12.7 \mathrm{~Hz}), 1.37(\mathrm{brs}, 1 \mathrm{H}), 1.18-1.27(\mathrm{~m}, 2 \mathrm{H}), 0.92(\mathrm{~d}, 3 \mathrm{H}, J=6.4 \mathrm{~Hz}) ;{ }^{13} \mathrm{C} \mathrm{NMR}\left(100 \mathrm{MHz}, \mathrm{CDCl}_{3}\right)$ (ppm): 170.6, 66.9, 66.7, 54.4, 54.1, 46.0, 41.9, 34.2, 31.0, 30.6, 21.8. Anal. Calcd for $\mathrm{C}_{13} \mathrm{H}_{24} \mathrm{~N}_{2} \mathrm{O}_{2}$ : C, 64.97; H, 10.07; N, 11.66; O, 13.31. Found: C, 64.87; H, 10.15; N, 11.64; O, 13.34.

\section{N-(tert-butyl)-3-(4-methylpiperidin-1-yl)propanamide (Table 4, entry 11)}

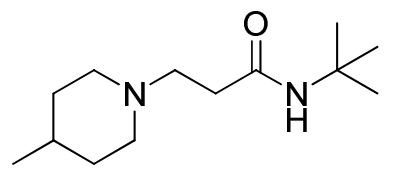

${ }^{1} \mathrm{H}$ NMR (400 MHz, $\left.\mathrm{CDCl}_{3}\right)(\mathrm{ppm}): 8.58(\mathrm{~s}, 1 \mathrm{H}), 2.92(\mathrm{~d}, 2 \mathrm{H}, J=11.6 \mathrm{~Hz}), 2.55(\mathrm{t}, 2 \mathrm{H}, J=6.0 \mathrm{~Hz})$, $2.28(\mathrm{t}, 2 \mathrm{H}, J=6.0 \mathrm{~Hz}), 1.95-2.00(\mathrm{~m}, 2 \mathrm{H}), 1.68(\mathrm{~d}, 2 \mathrm{H}, J=13.2 \mathrm{~Hz}), 1.40(\mathrm{~m}, 1 \mathrm{H}), 1.33(\mathrm{~s}, 9 \mathrm{H})$, 1.16-1.19(m, 2H), 0.93(d, 3H, $J=6.8 \mathrm{~Hz}) ;{ }^{13} \mathrm{C} \mathrm{NMR}\left(100 \mathrm{MHz}, \mathrm{CDCl}_{3}\right)(\mathrm{ppm}): 172.0,54.4,53.1$, 
50.3, 34.4, 33.1, 30.7, 28.9, 21.8. Anal. Calcd for $\mathrm{C}_{13} \mathrm{H}_{26} \mathrm{~N}_{2} \mathrm{O}$ : C, 68.98; H, 11.58; N, 12.38; O, 7.07.

Found: C, 68.84; H, 11.69; N, 12.32; O, 7.15.

N-(hydroxymethyl)-3-(4-methylpiperidin-1-yl)propanamide (Table 4, entry 12)<smiles>CC1CCN(CCC(=O)NCO)CC1</smiles>

${ }^{1} \mathrm{H}$ NMR (400 MHz, $\left.\mathrm{CDCl}_{3}\right)(\mathrm{ppm}): 8.98(\mathrm{~s}, 1 \mathrm{H}), 4.69(\mathrm{~d}, 1 \mathrm{H}, J=6.0 \mathrm{~Hz}), 3.70(\mathrm{~d}, 1 \mathrm{H}, J=3.2 \mathrm{~Hz})$, $3.58(\mathrm{~d}, 1 \mathrm{H}, J=3.2 \mathrm{~Hz}), 3.09(\mathrm{~d}, 2 \mathrm{H}, J=11.6 \mathrm{~Hz}), 2.82(\mathrm{~s}, 2 \mathrm{H}), 2.52(\mathrm{~s}, 2 \mathrm{H}), \quad 2.19-2.24(\mathrm{~m}, 2 \mathrm{H}), 1.96(\mathrm{~s}$, 1H), $1.68(\mathrm{~d}, 2 \mathrm{H}, J=13.2 \mathrm{~Hz}), 1.44(\mathrm{~s}, 1 \mathrm{H}), 1.30-1.43(\mathrm{~m}, 2 \mathrm{H}), 0.92(\mathrm{q}, 3 \mathrm{H}, J=6.4 \mathrm{~Hz}) ;{ }^{13} \mathrm{C}$ NMR $(100$ $\mathrm{MHz}, \mathrm{CDCl}_{3}$ ) (ppm): 173.2, 63.7, 61.4, 53.4, 53.0, 33.3, 32.9, 31.9, 29.9, 21.4. Anal. Calcd for $\mathrm{C}_{10} \mathrm{H}_{20} \mathrm{~N}_{2} \mathrm{O}_{2}$ : C, 59.97; H, 10.07; N, 13.99; O, 15.98. Found: C, C, 59.88; H, 10.17; N, 13.94; O, 16.01 .

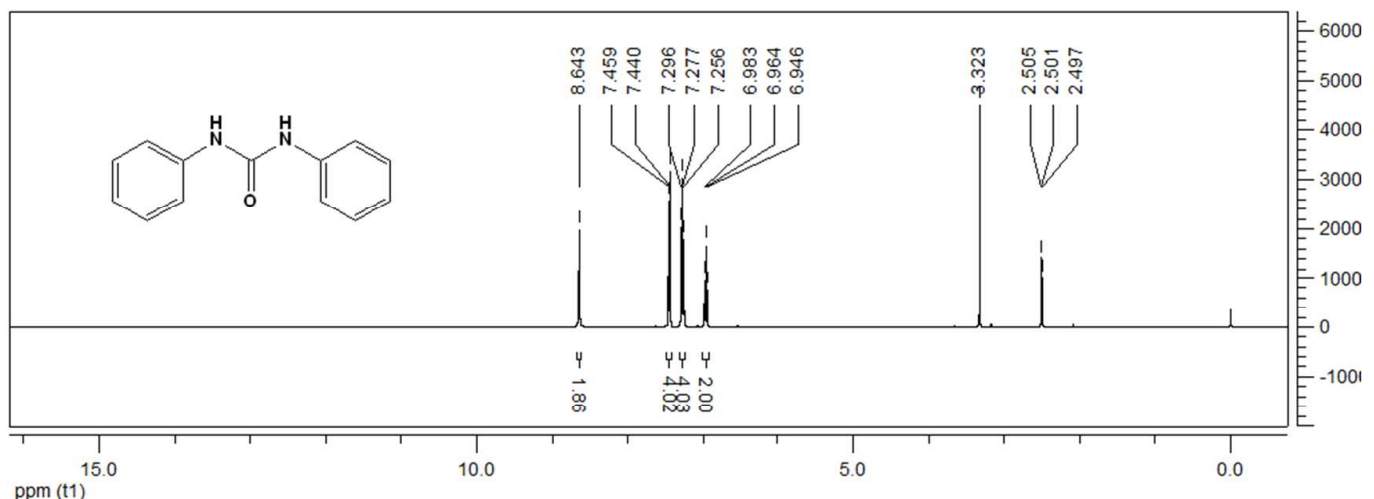



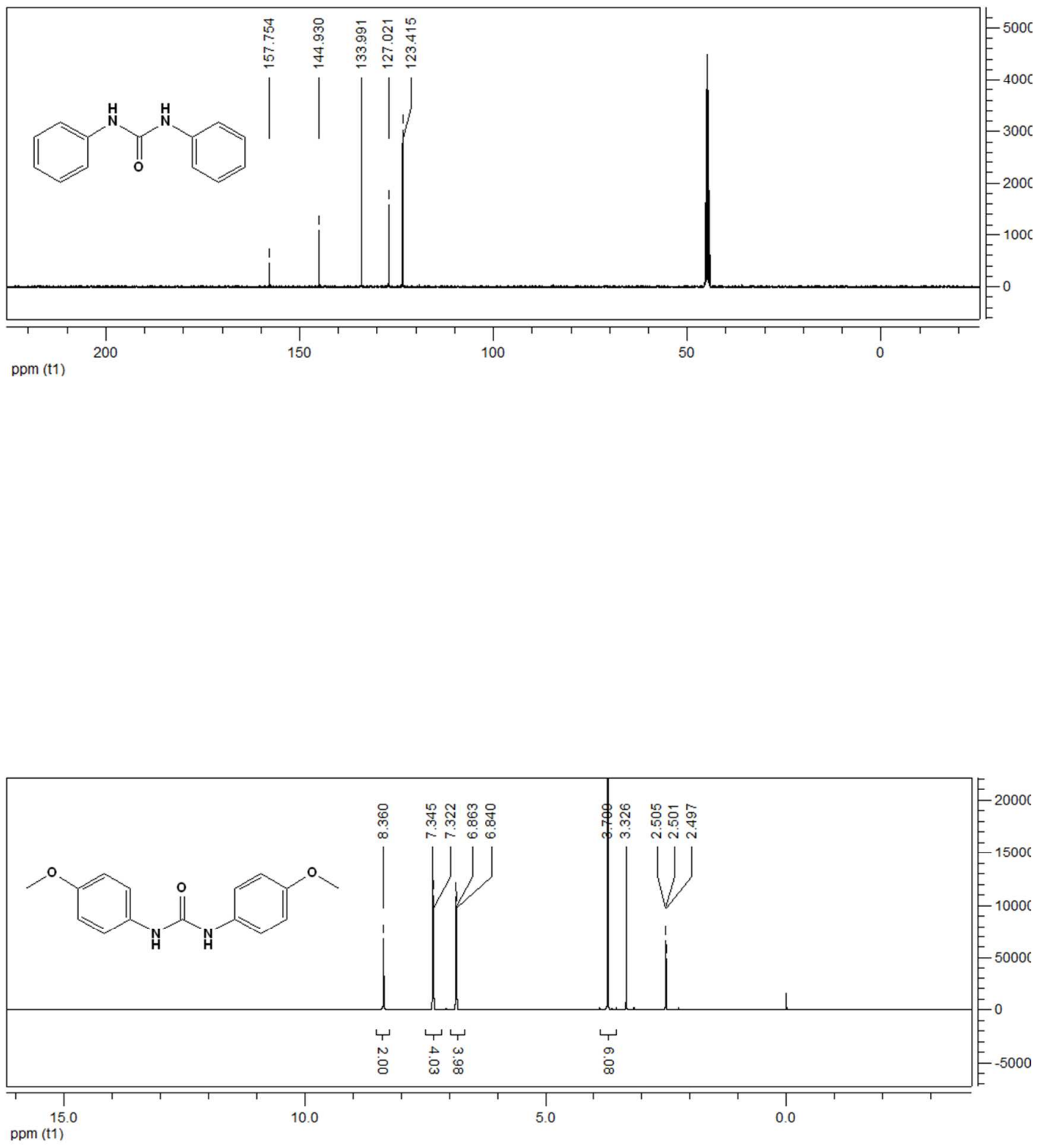

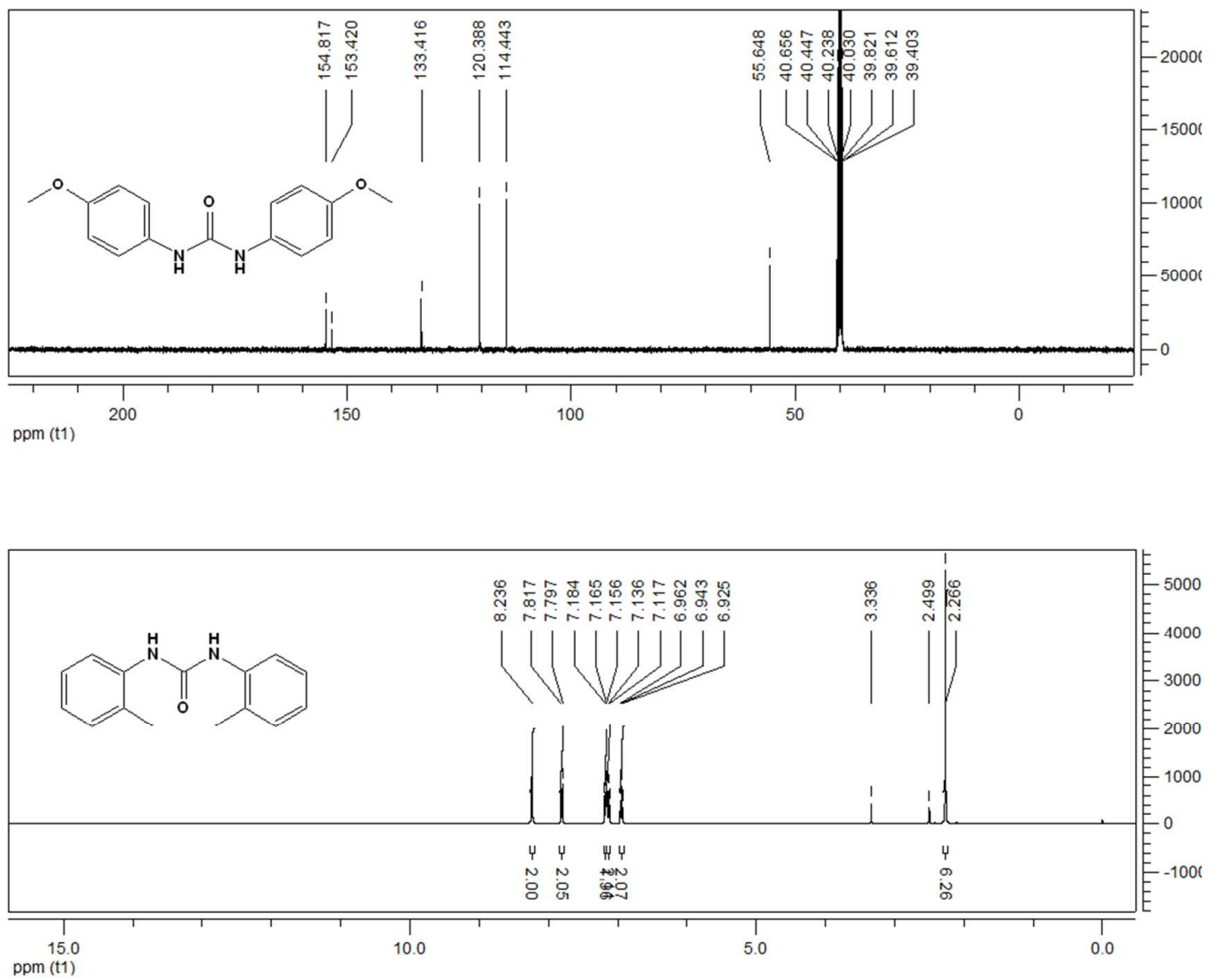

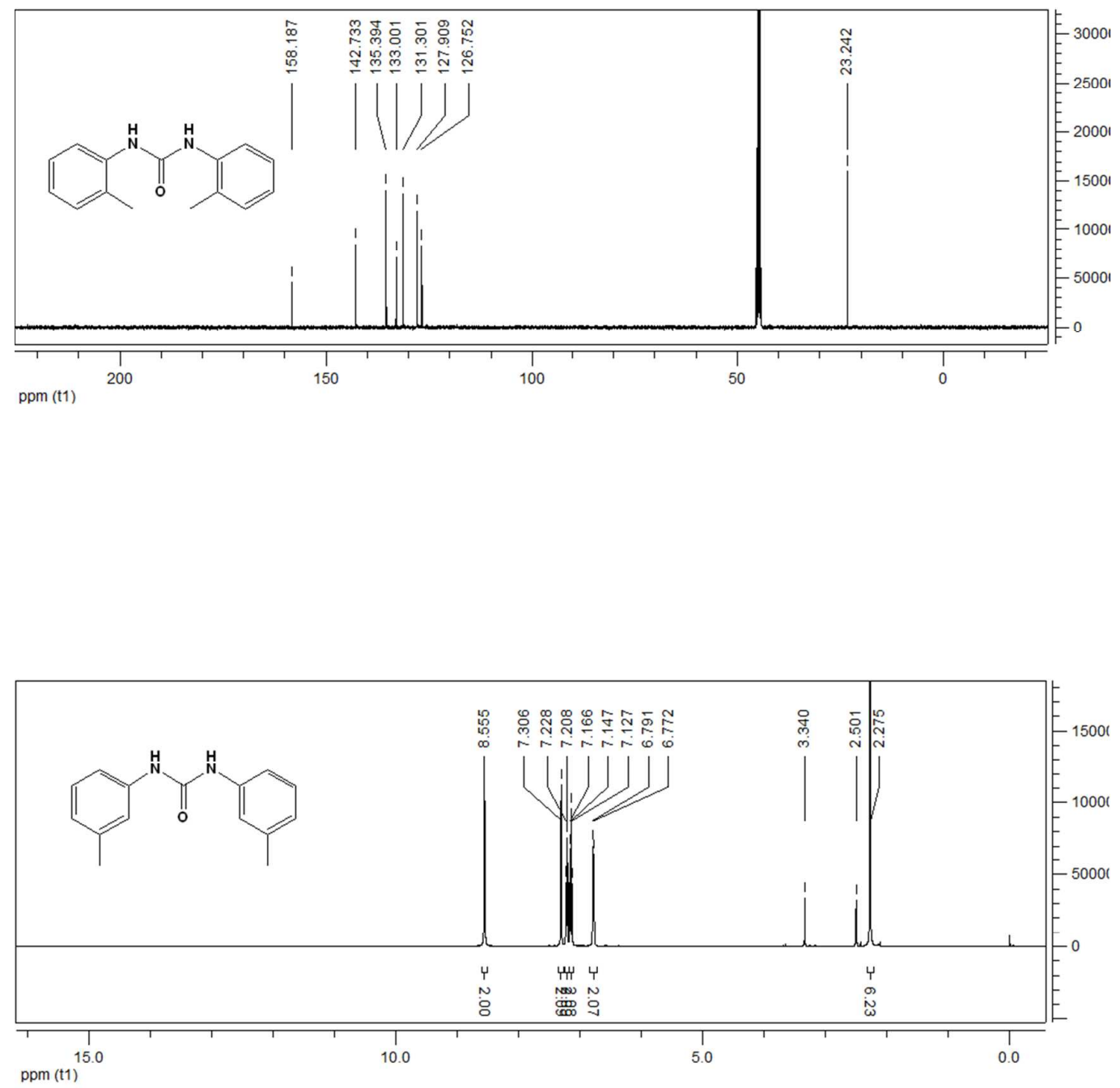

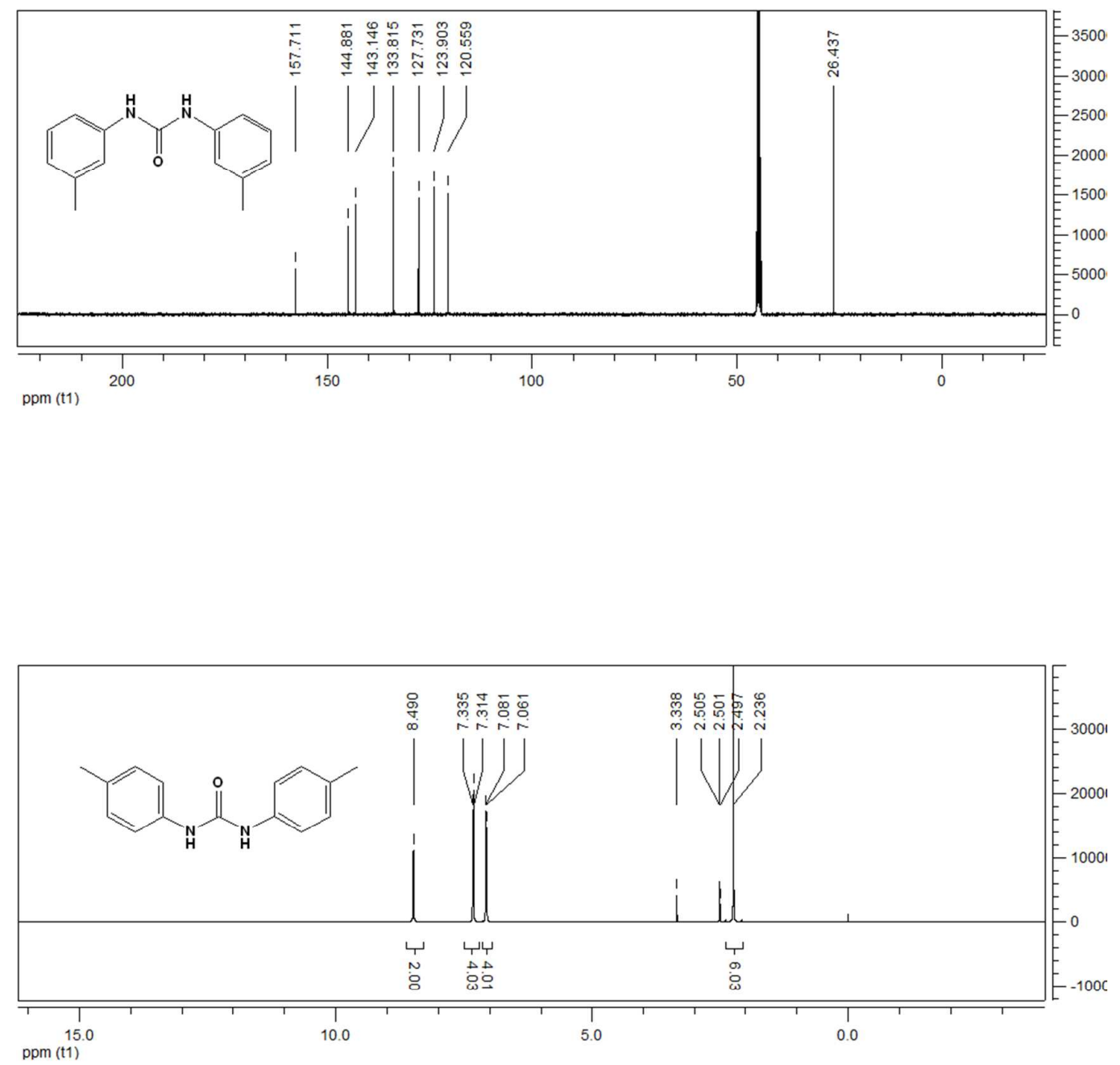


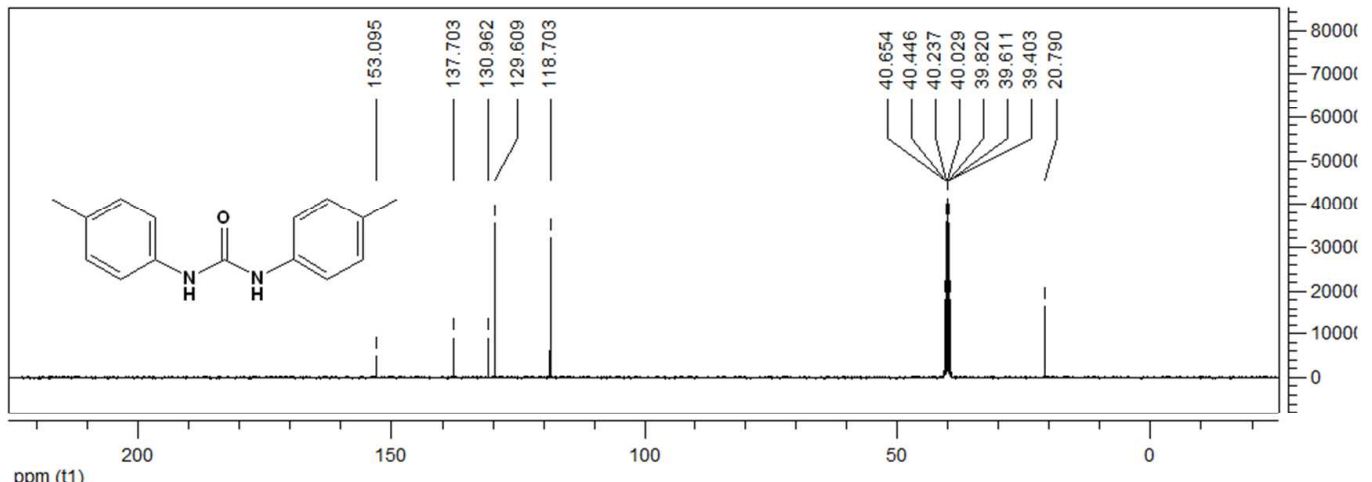

ppm (t1)
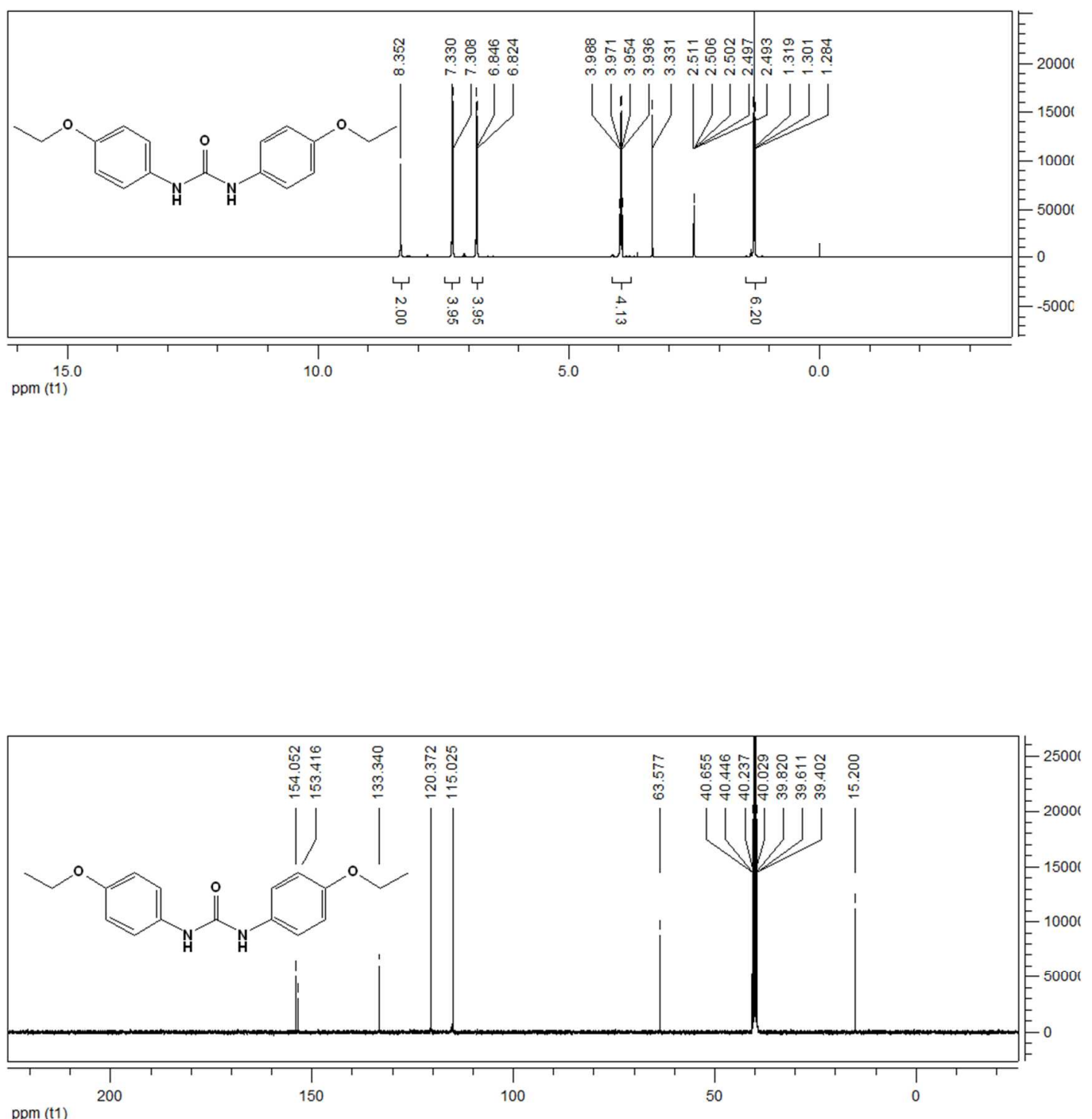

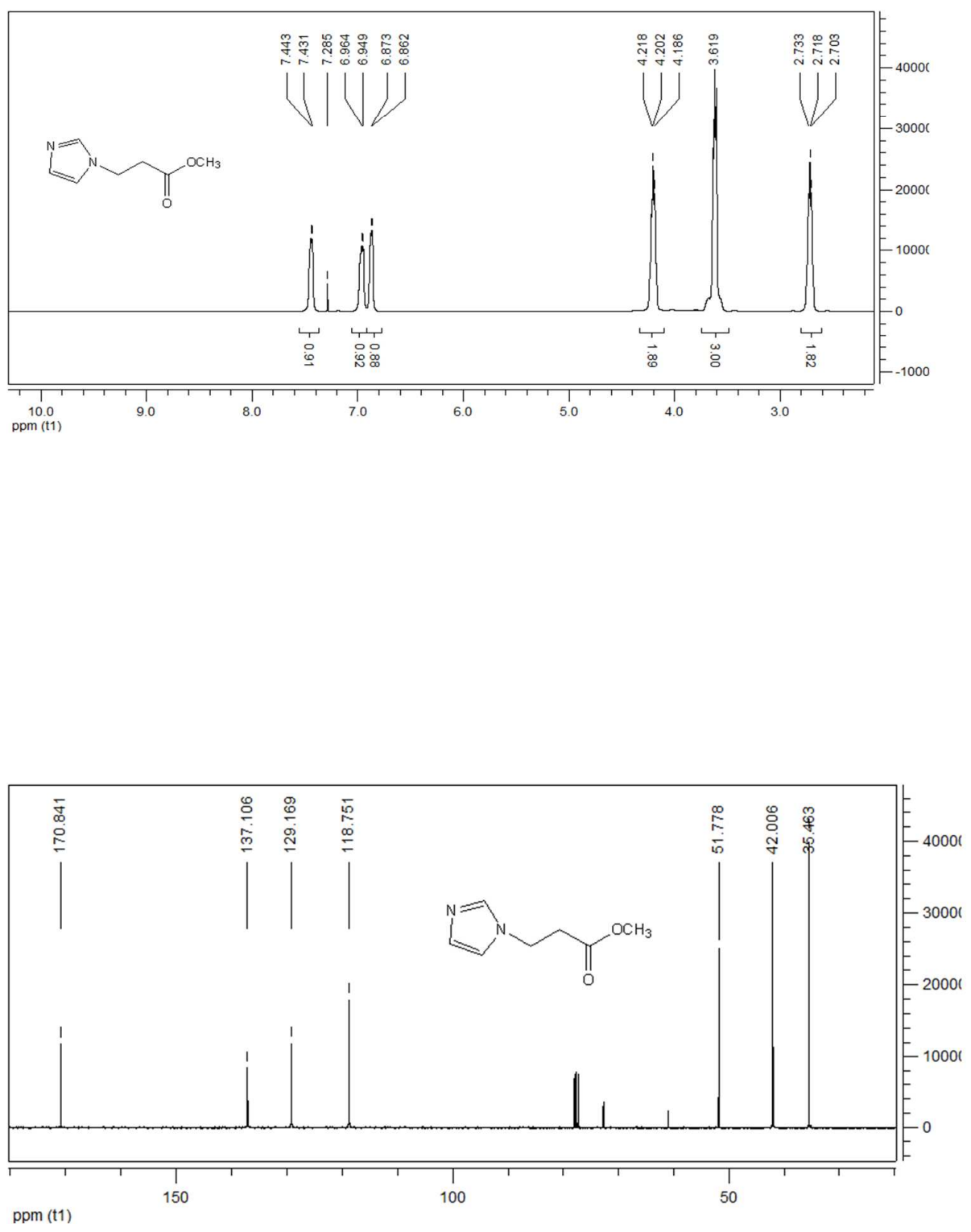

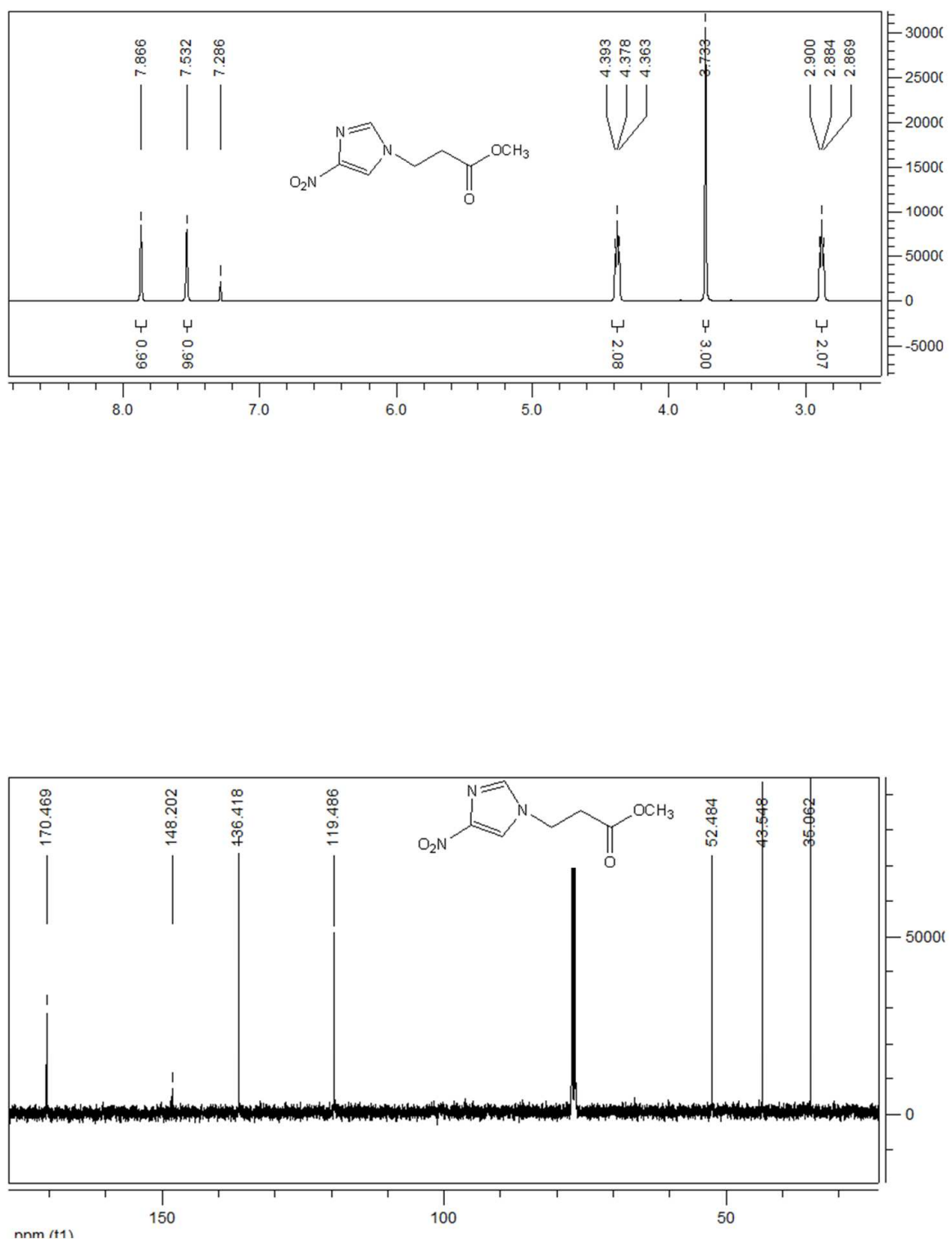


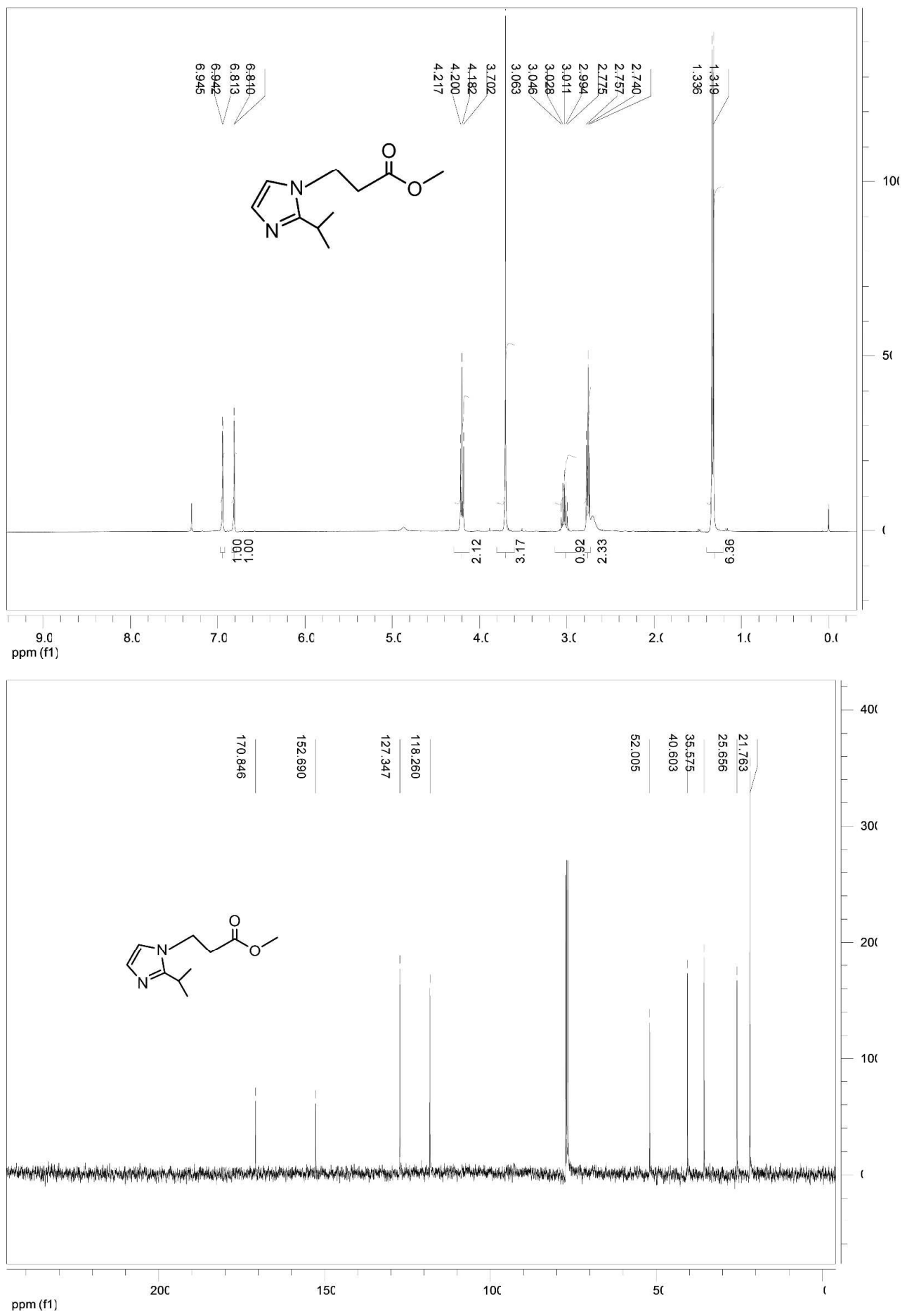



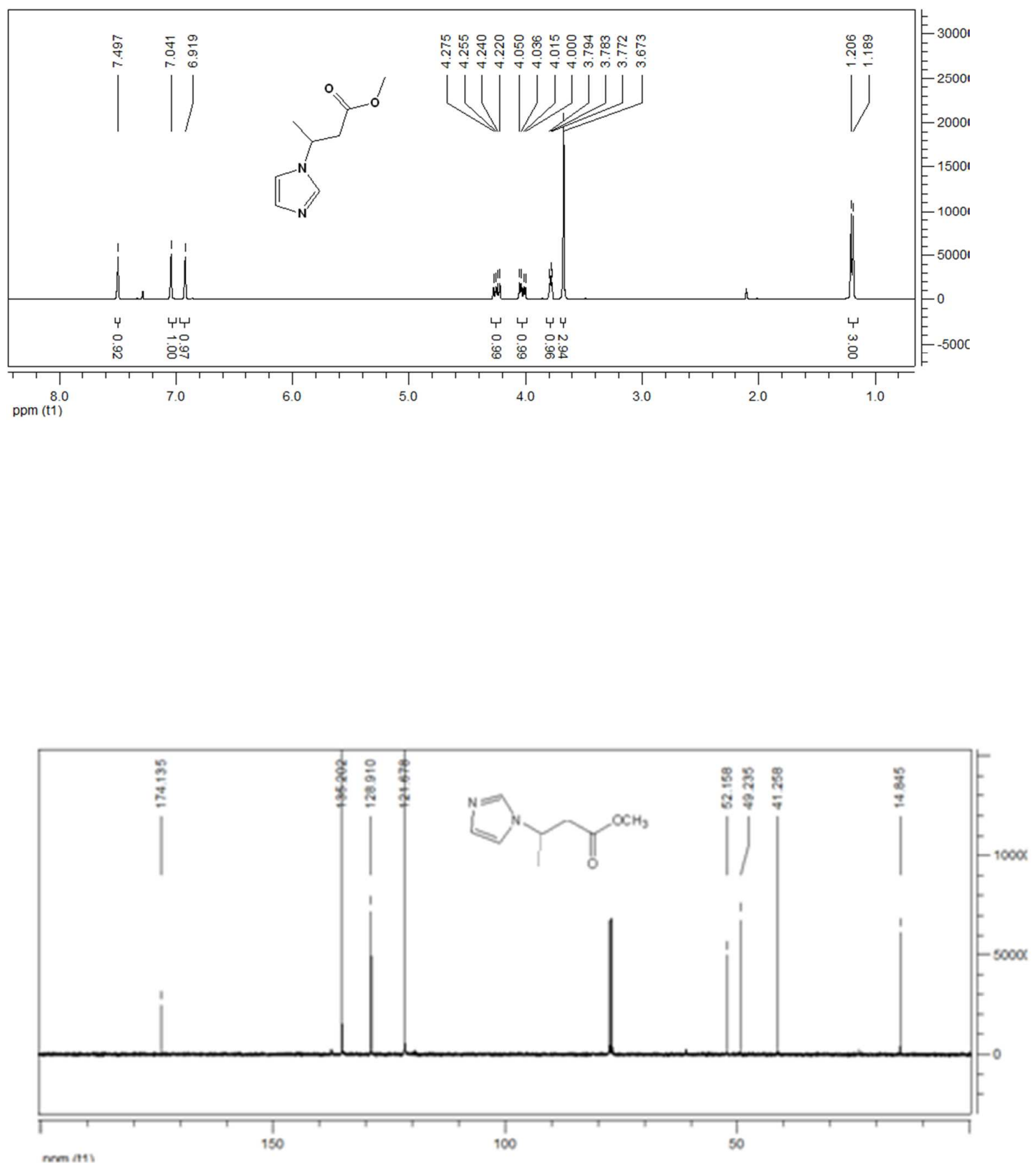


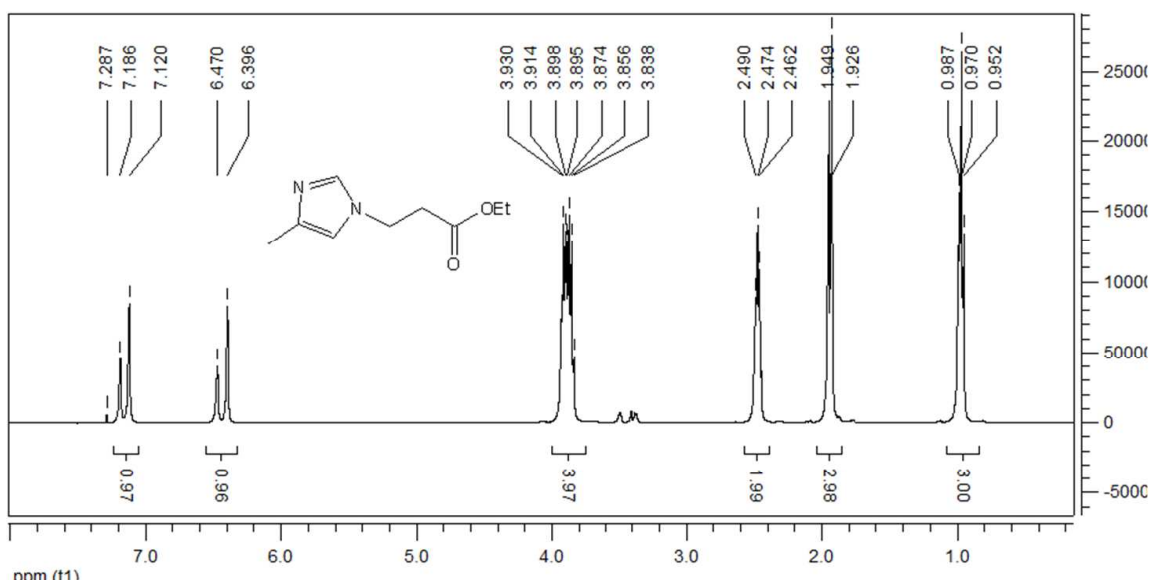

ppm (t1)

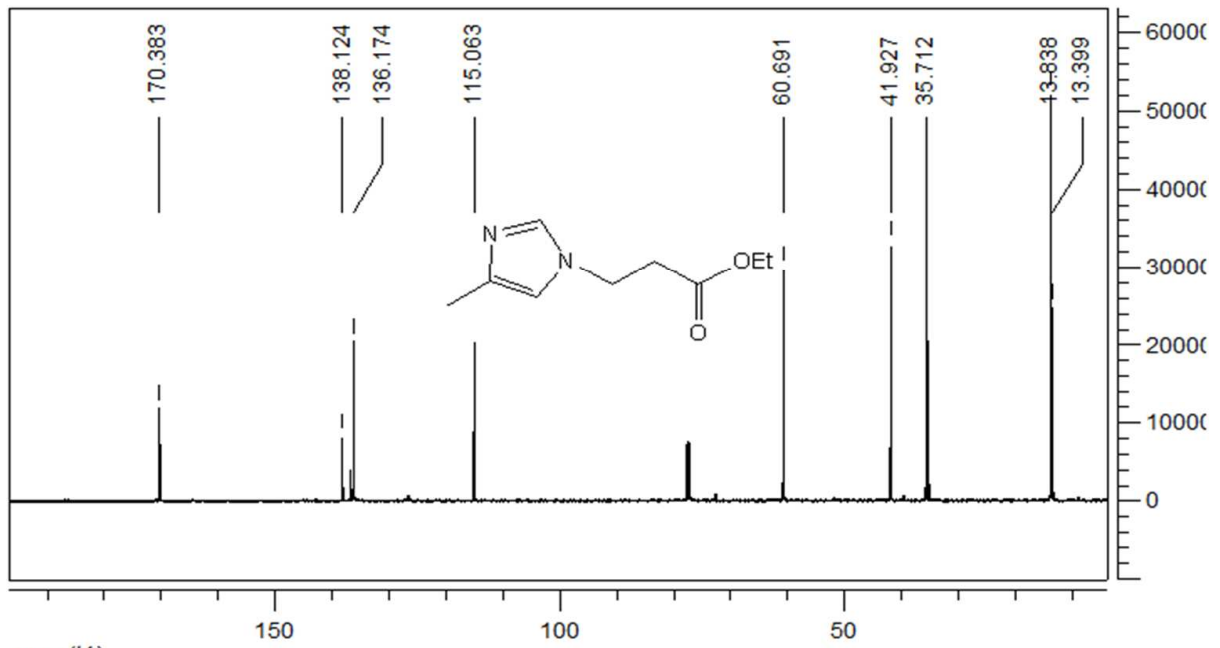

ppm (t1) 

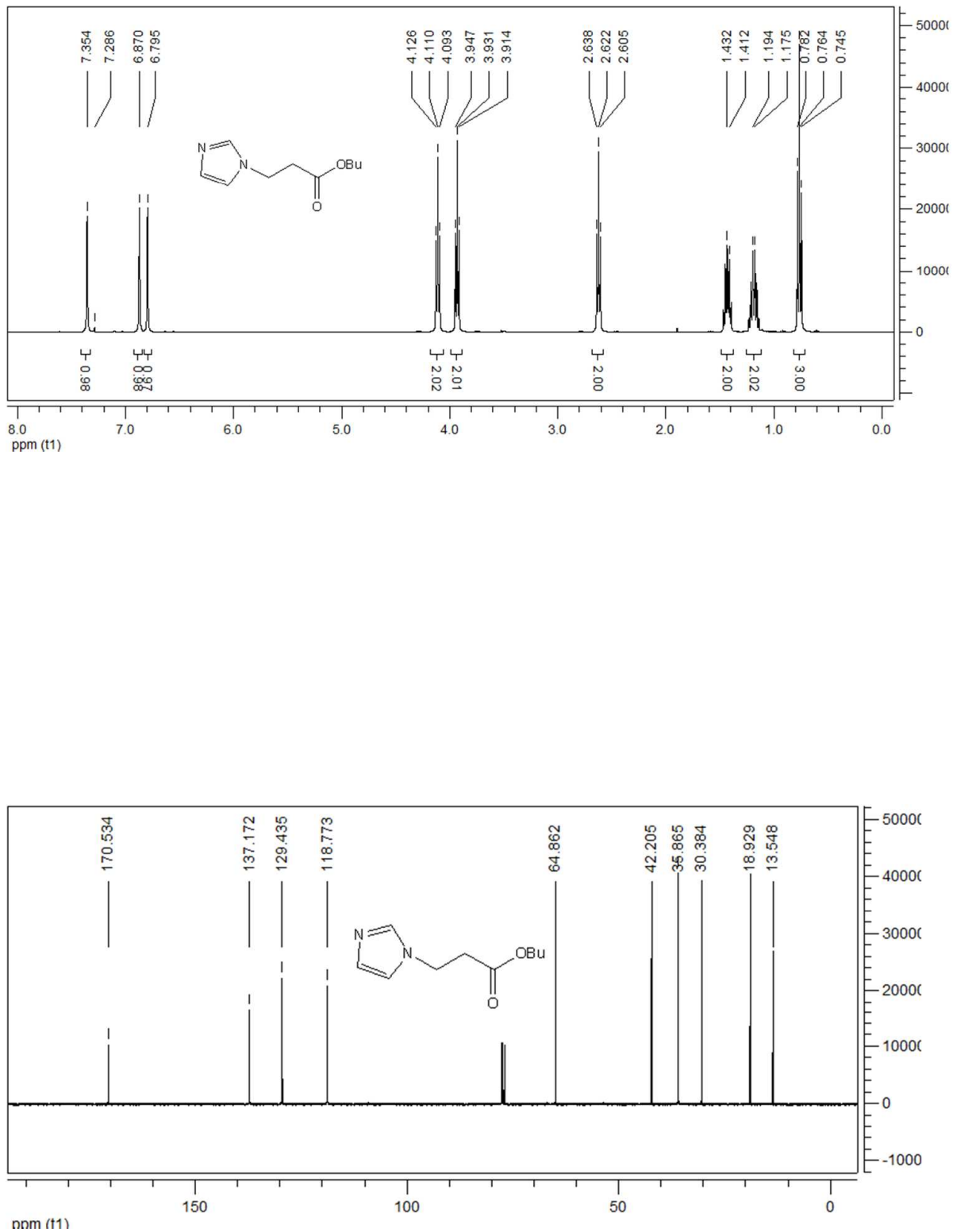

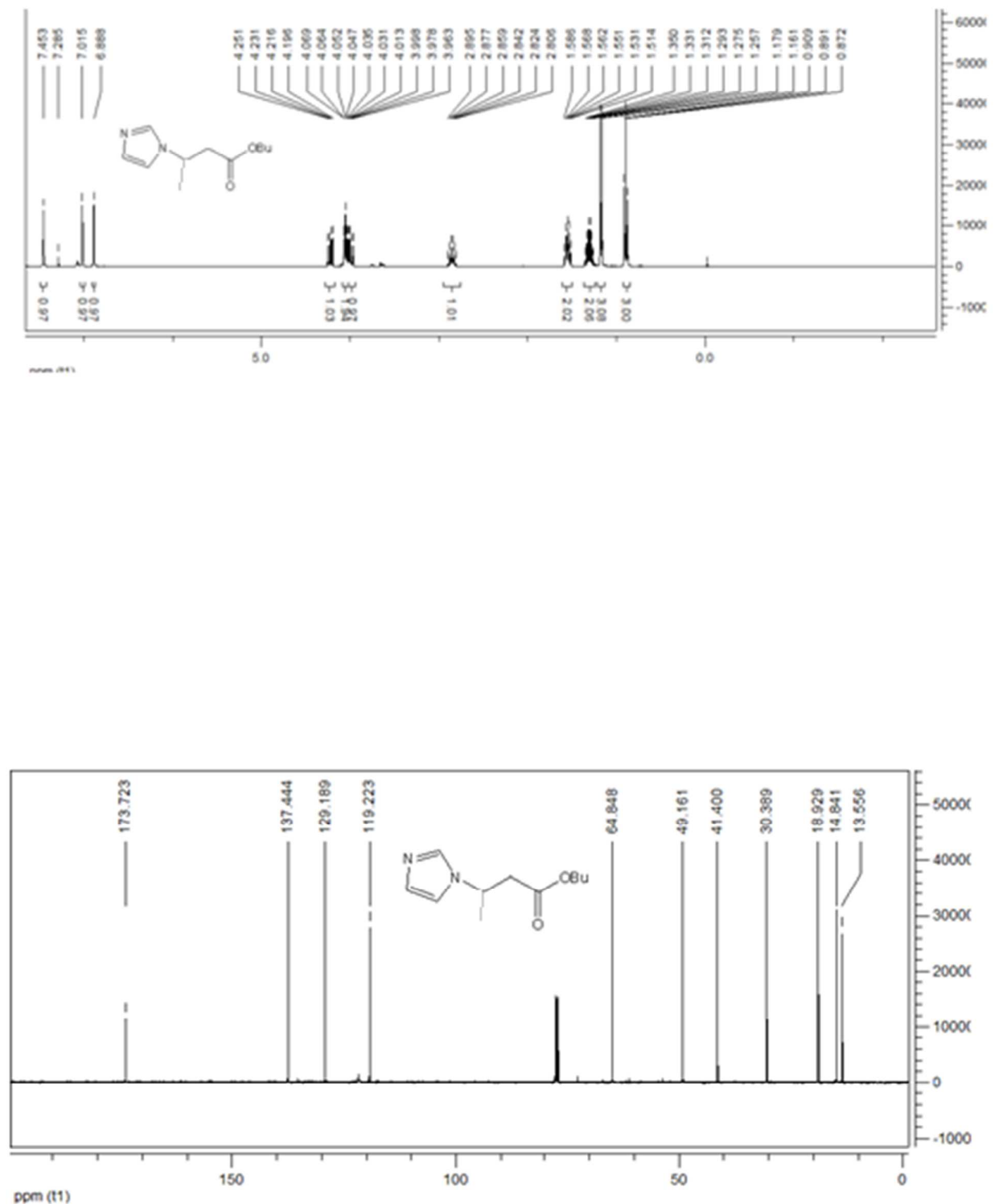


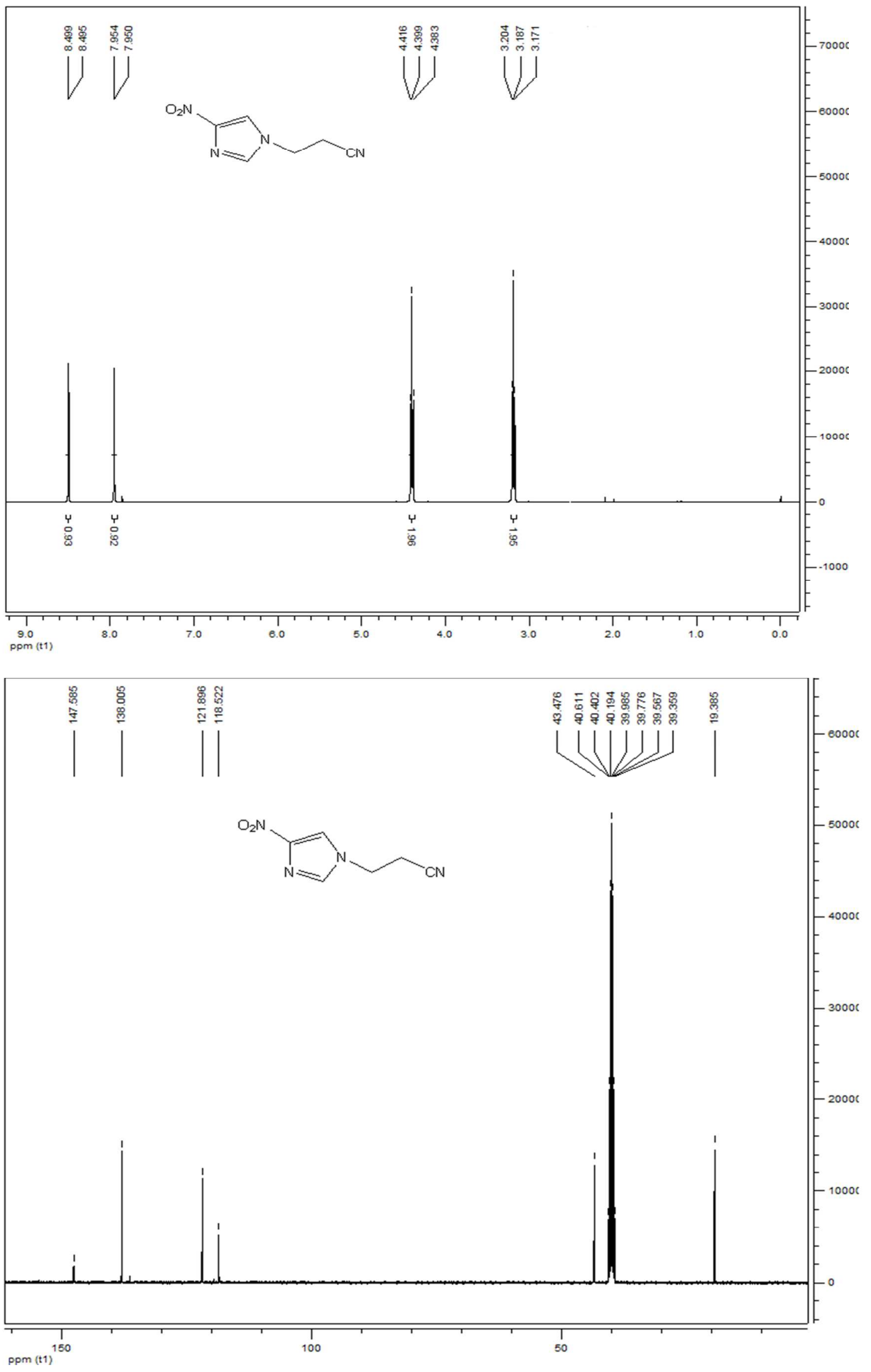



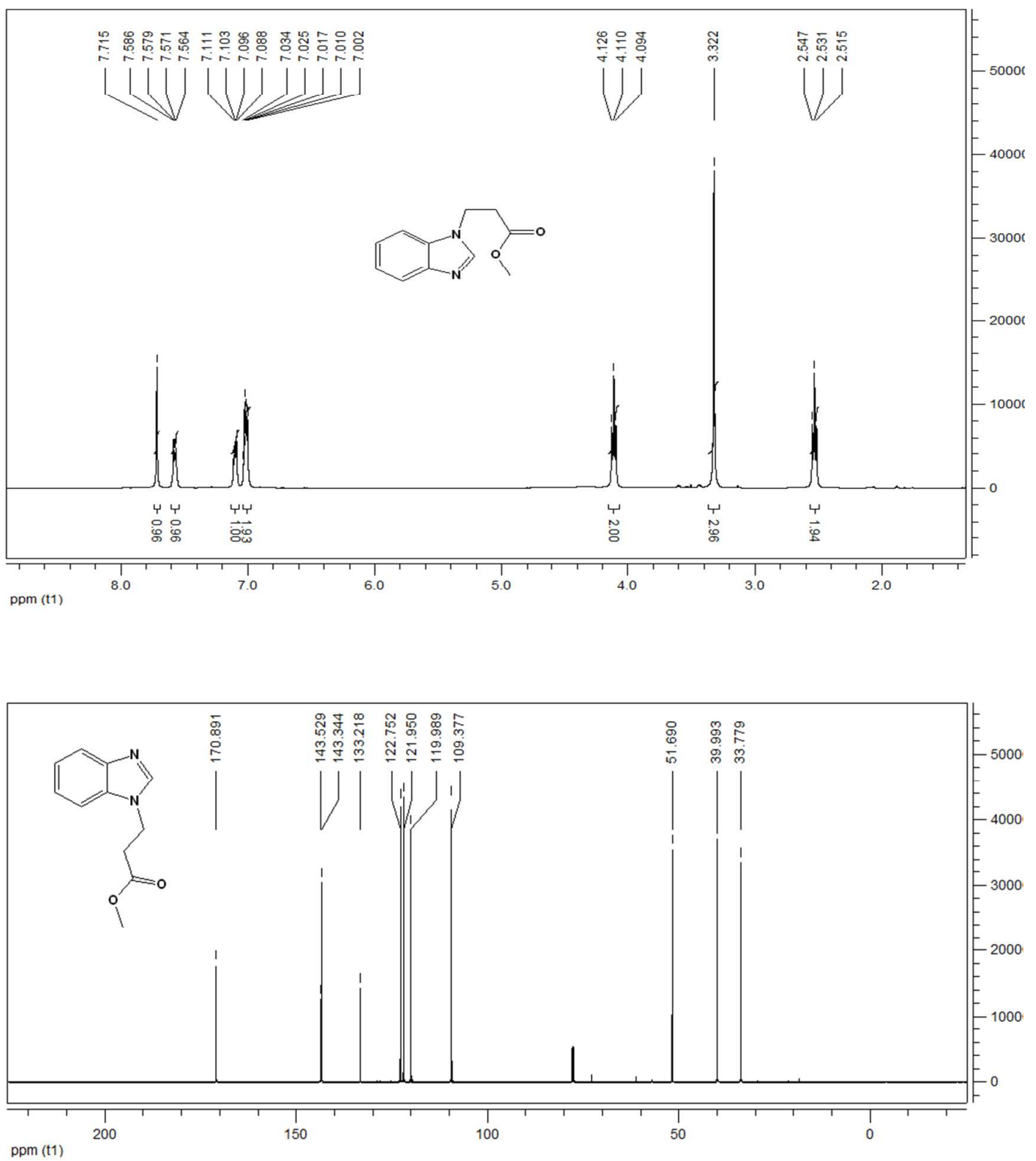

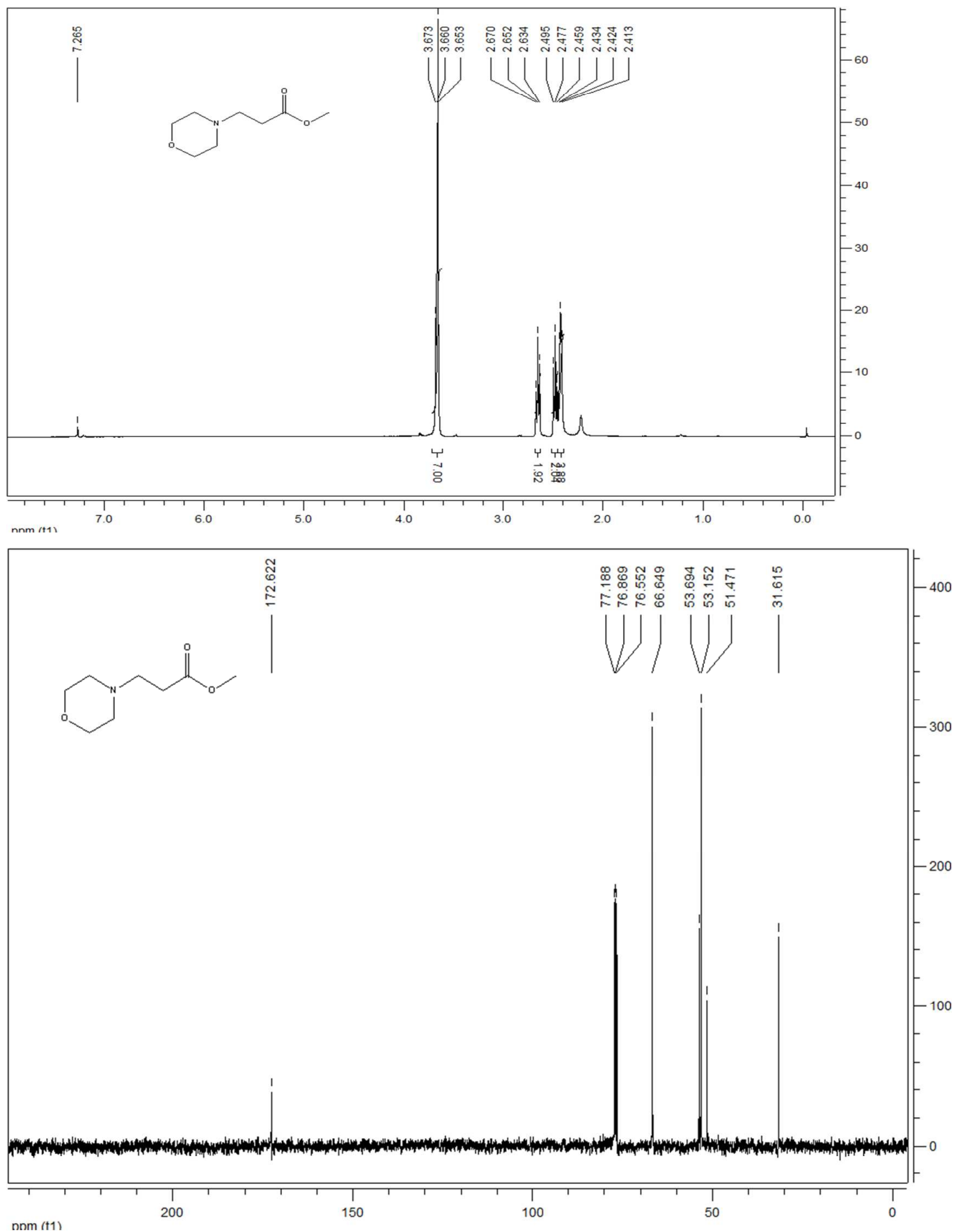

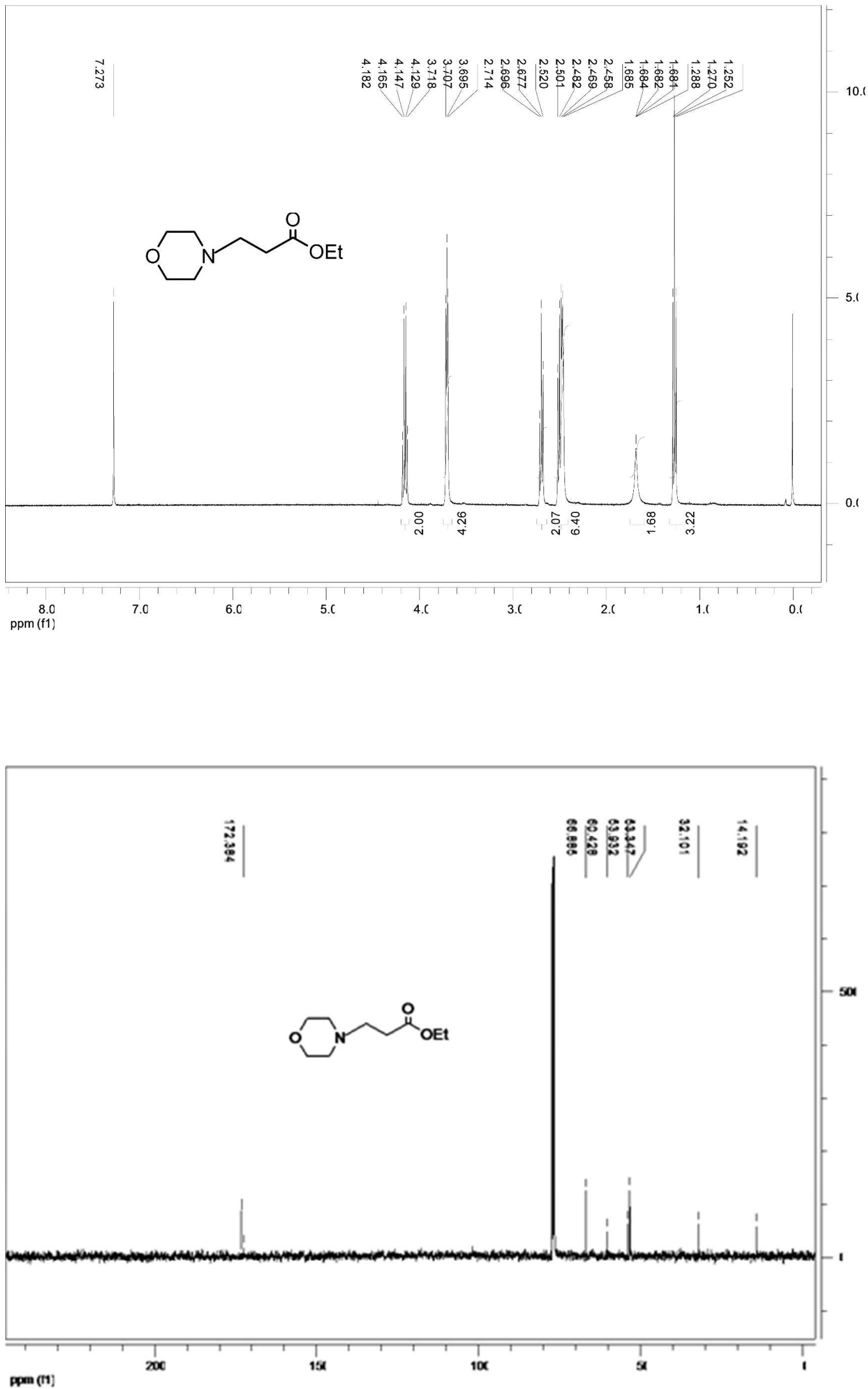


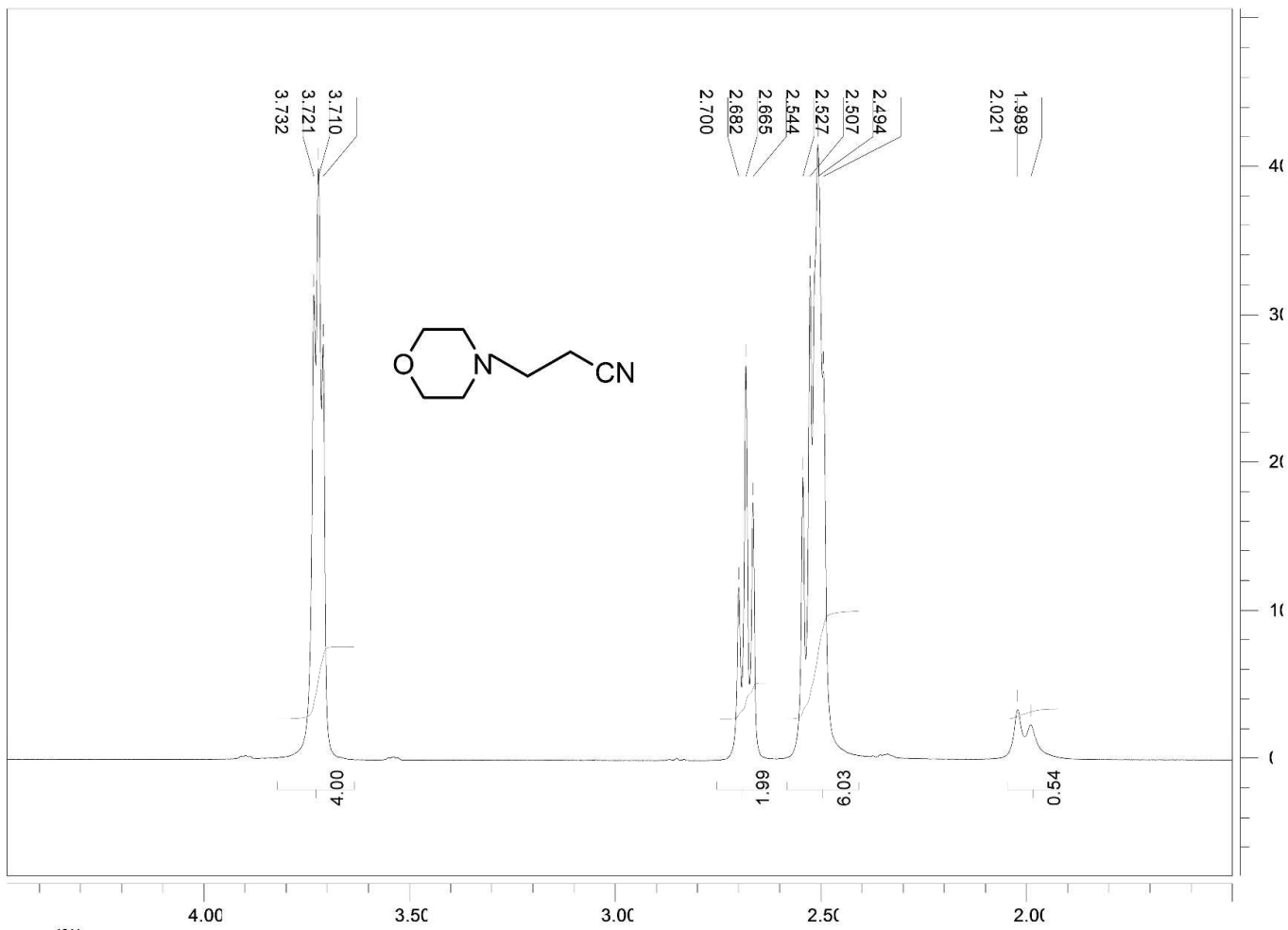

ppm (f1)

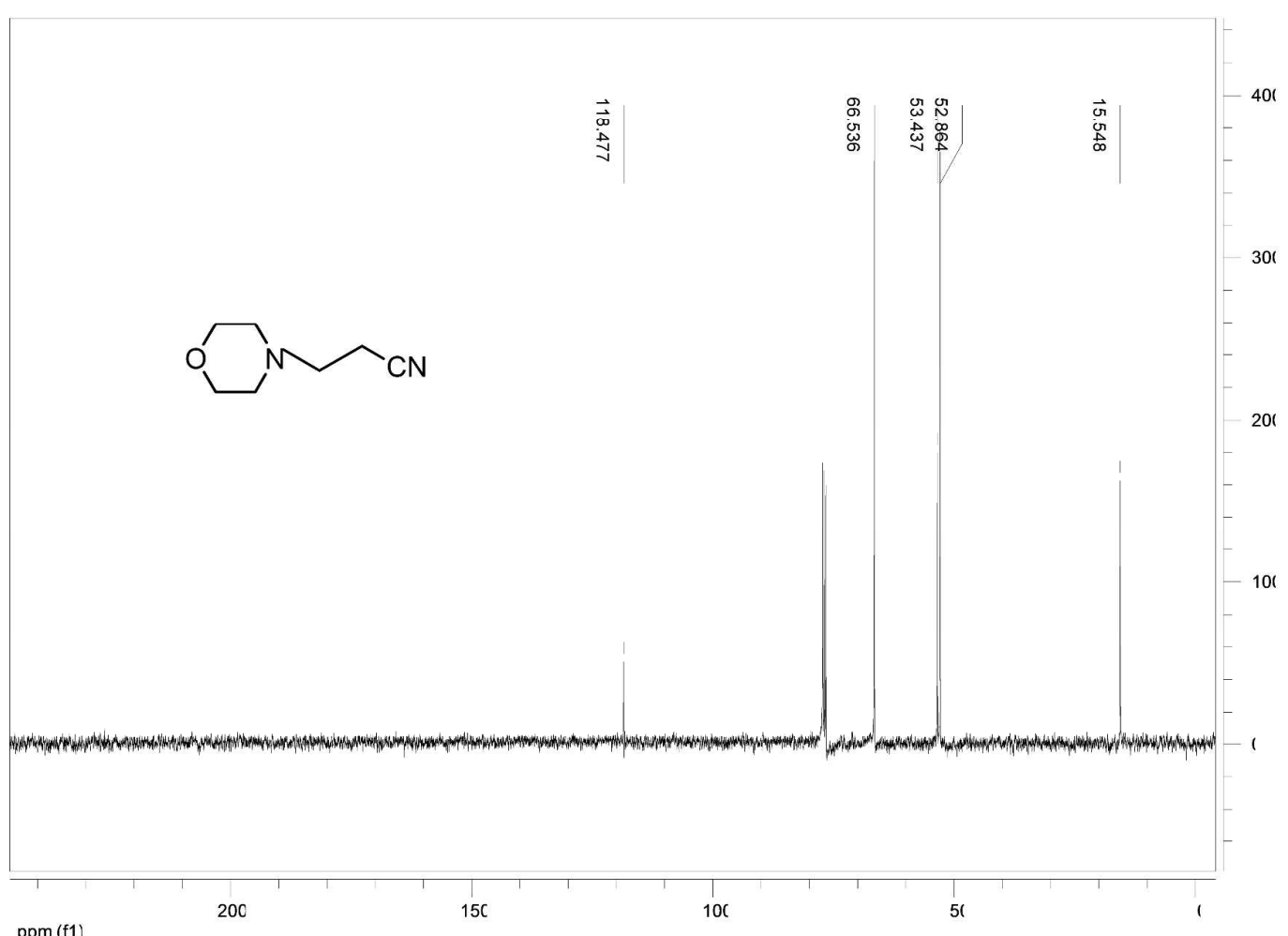

ppm (f1) 

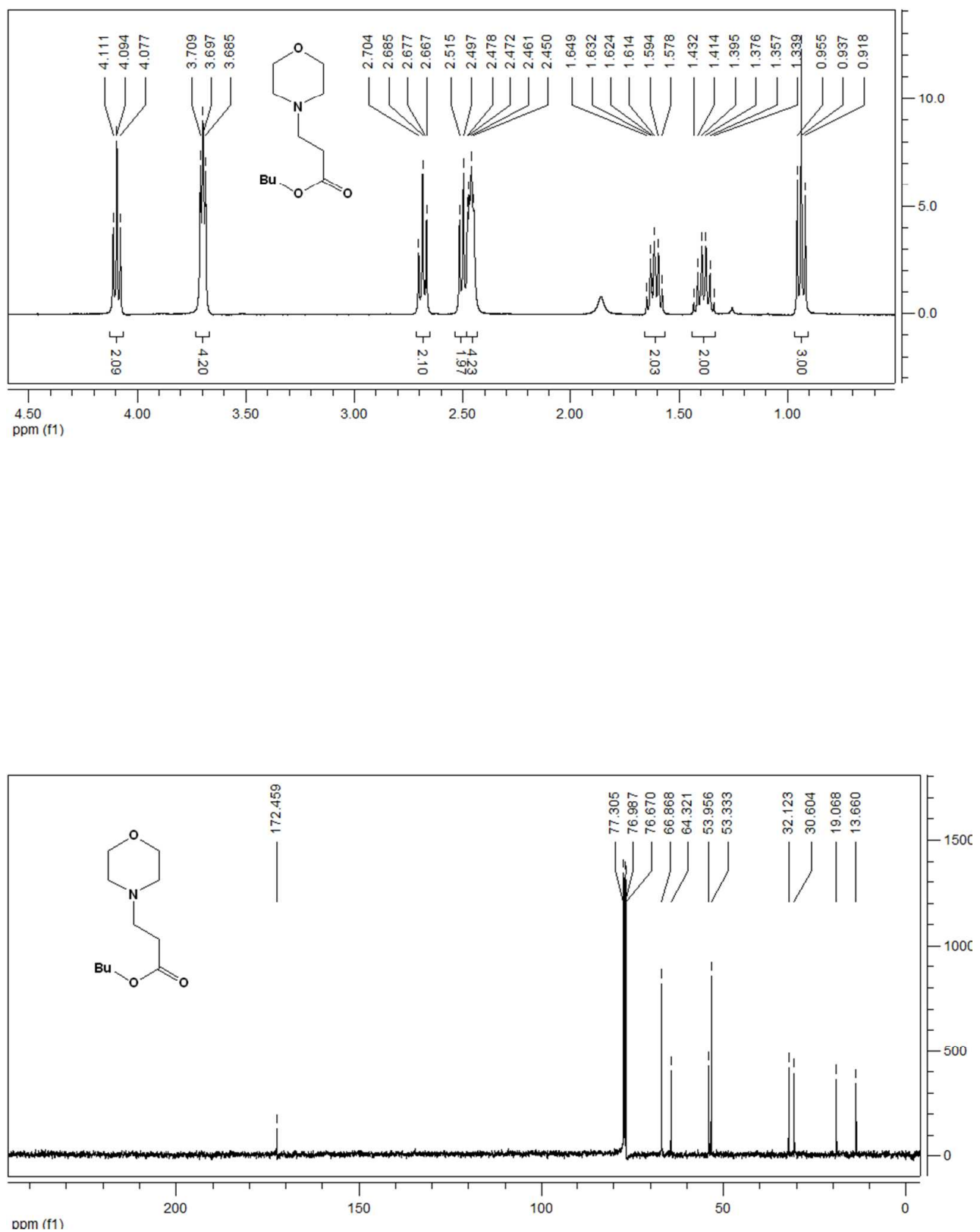


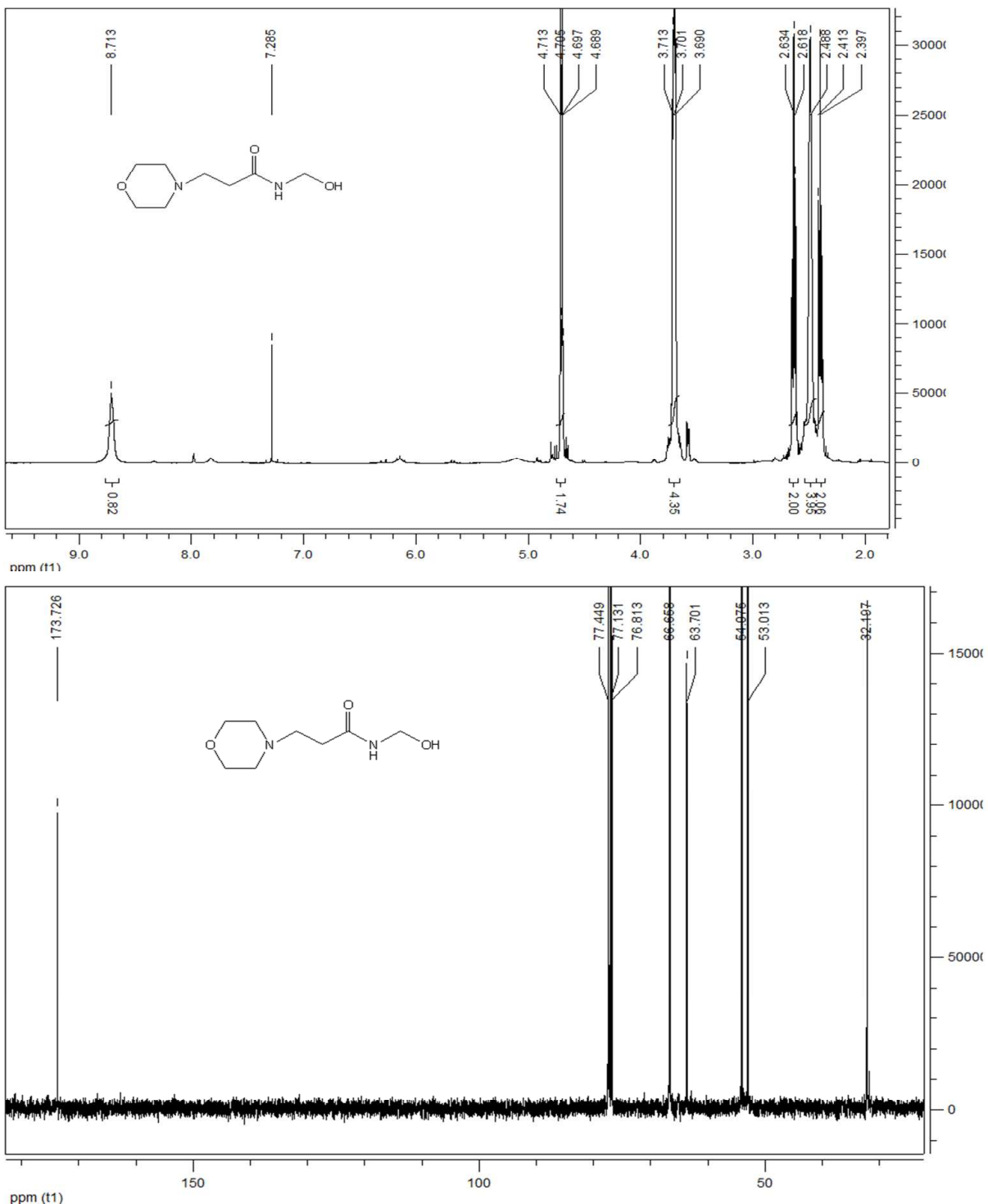




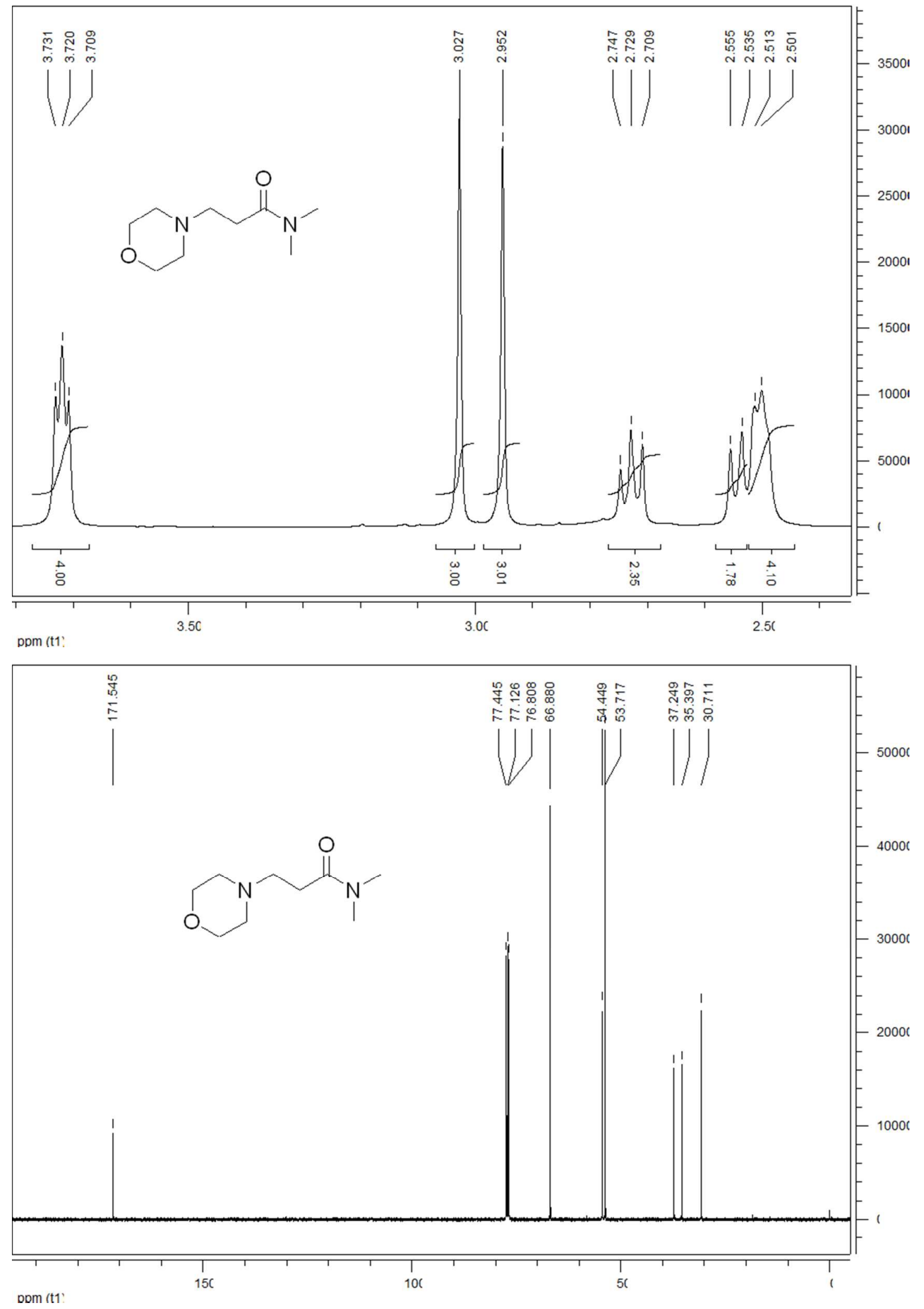




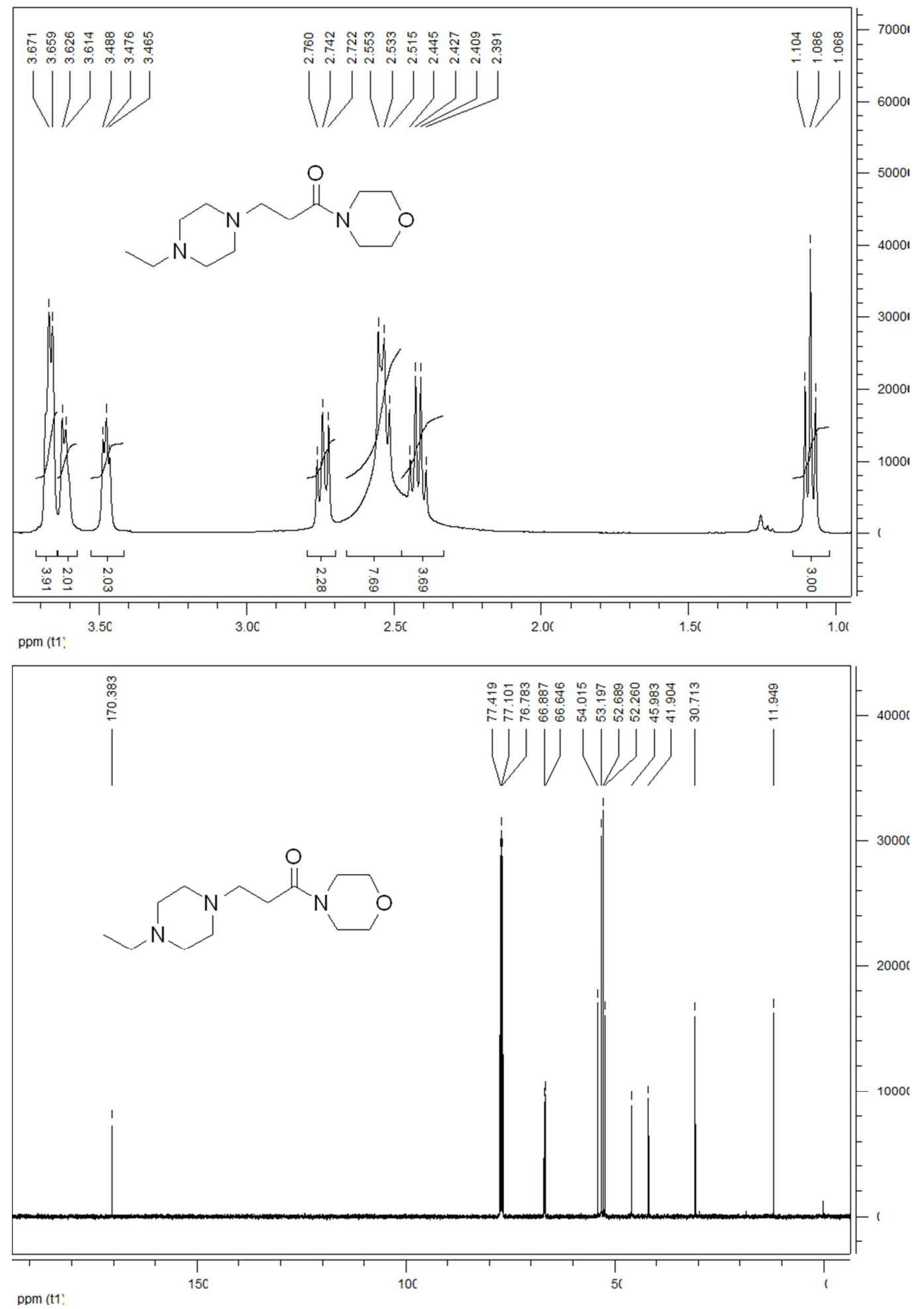



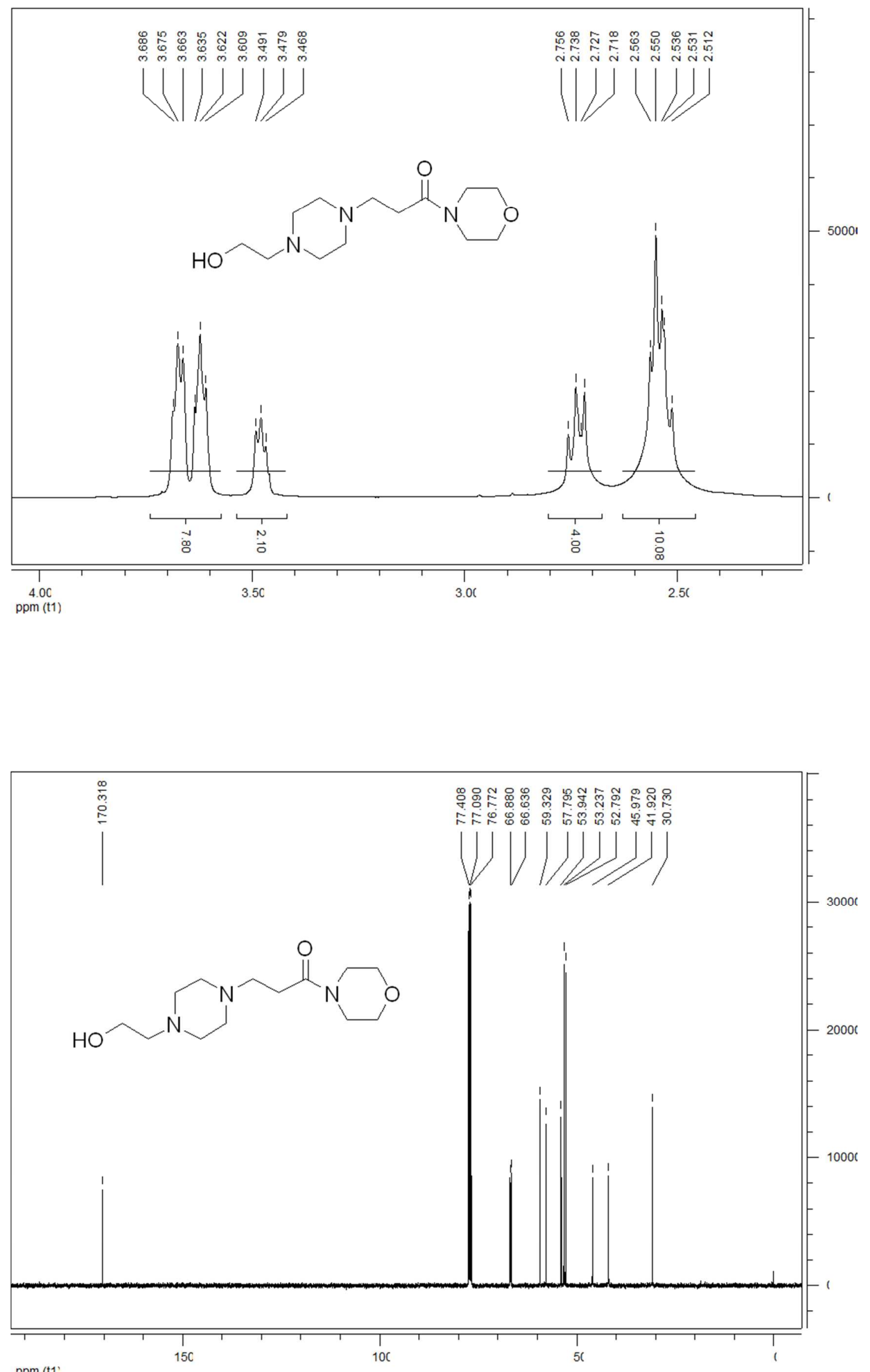

ppm (t1) 

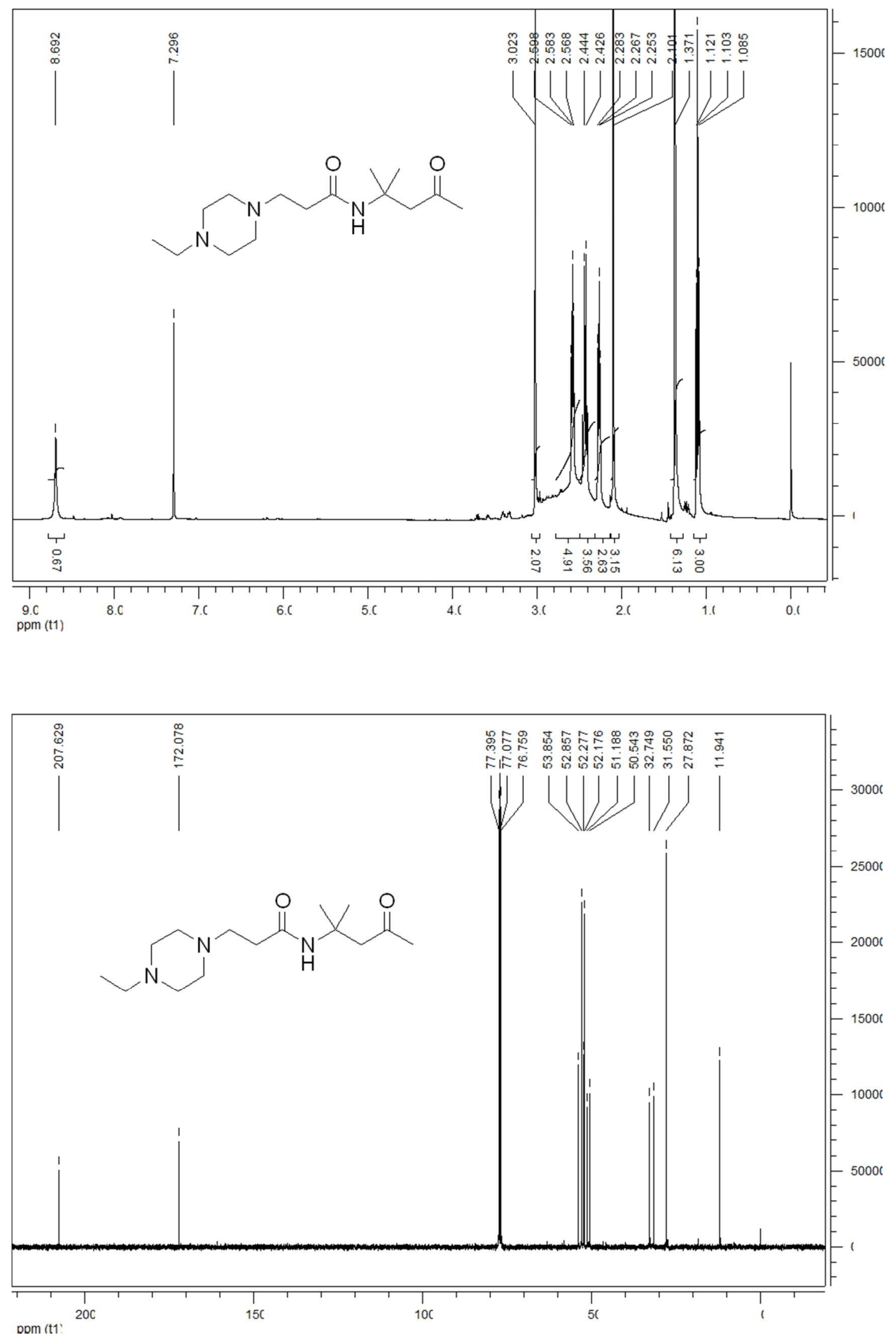


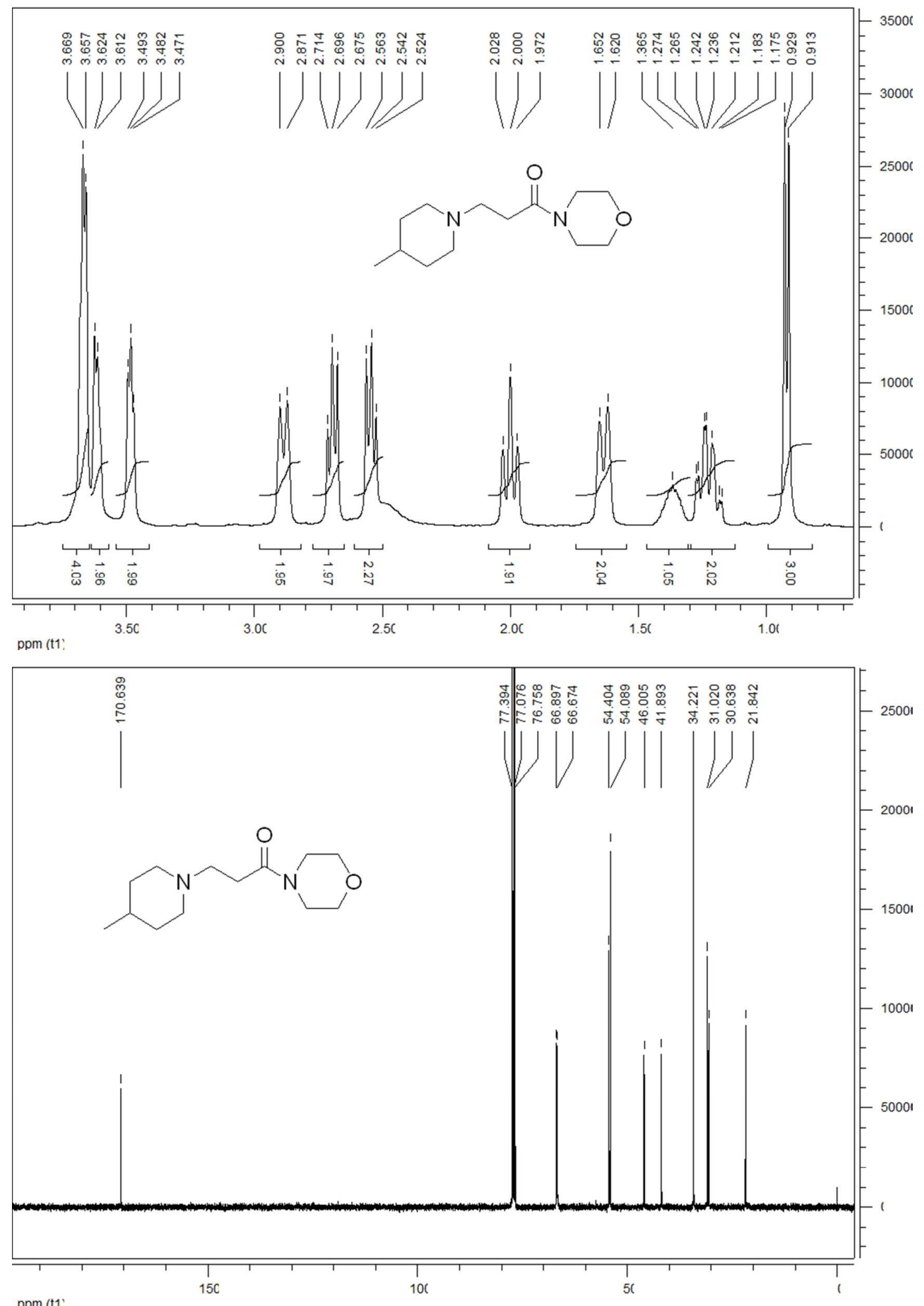

ppm (t1) 


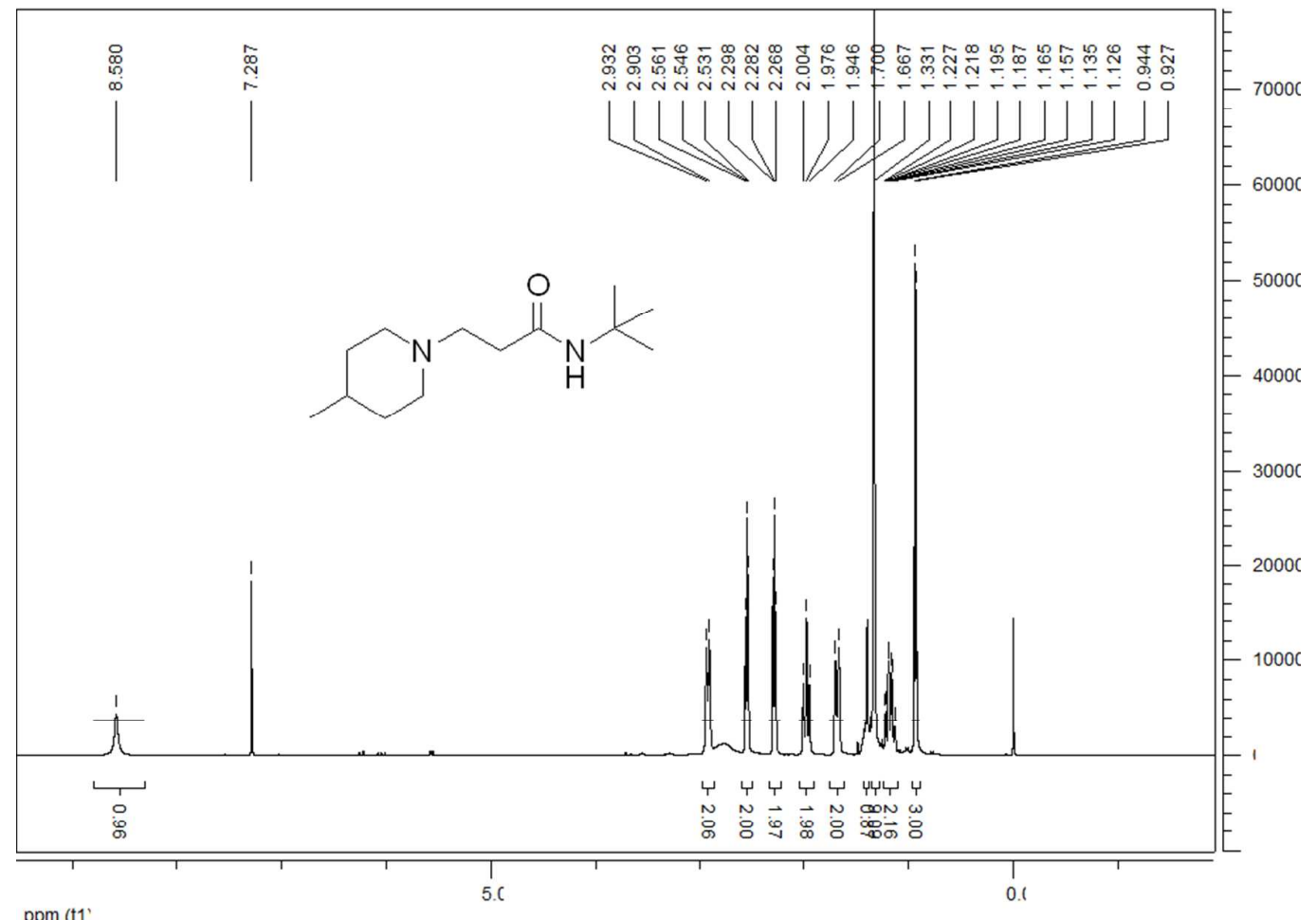

ppm (t1)

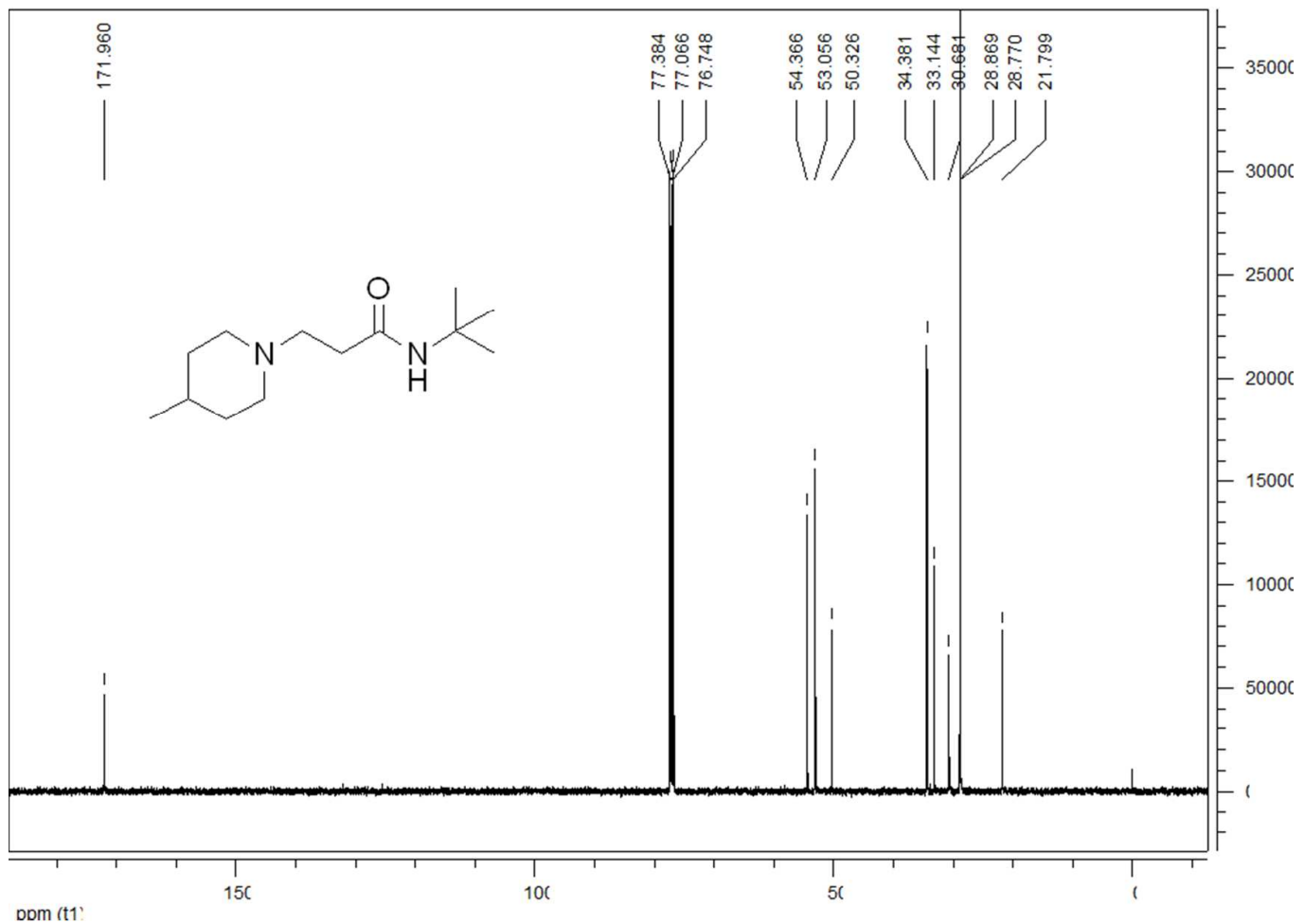




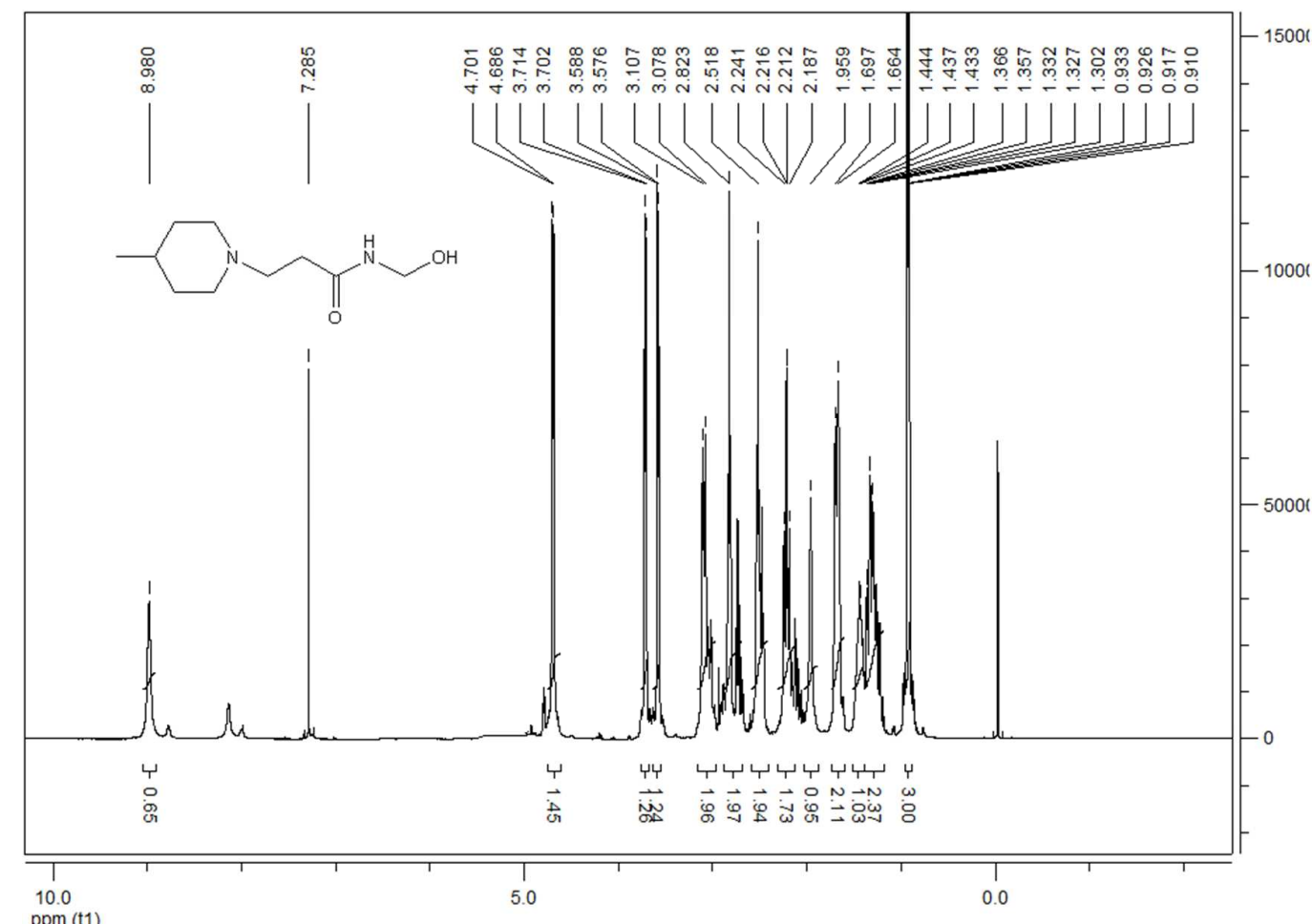

ppm (t1)
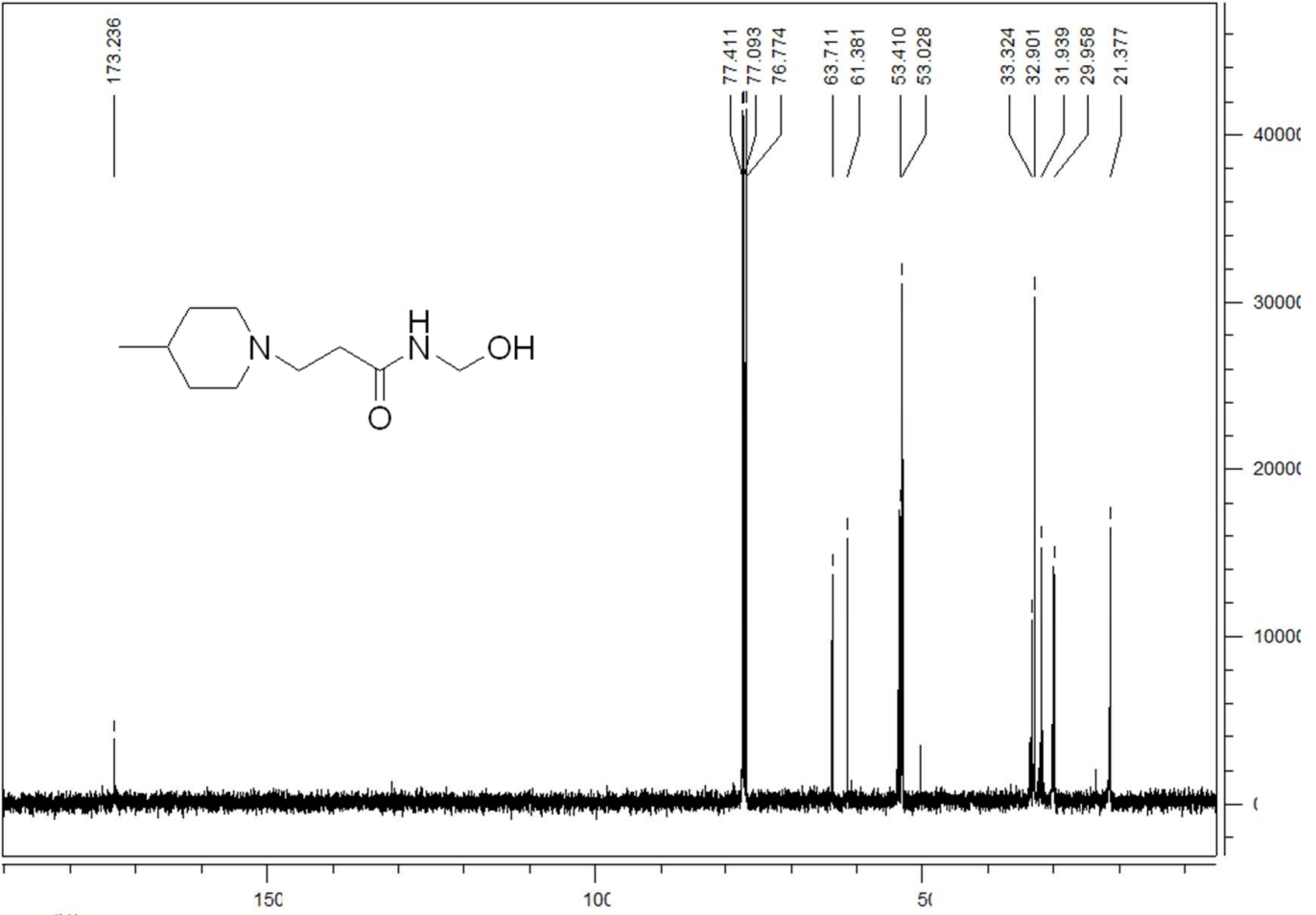

ppm (t1) 